\title{
A suture-related accretionary wedge formed in the Neoproterozoic Araçuaí orogen (SE Brazil) during Western Gondwanaland assembly
}

\author{
Eliza Peixoto $^{\text {a,* }}$, A.C. Pedrosa-Soares ${ }^{\text {a,1 }}$, F.F. Alkmim ${ }^{\text {b,1 }}$, I.A. Dussin ${ }^{\text {a,1 }}$ \\ a Universidade Federal de Minas Gerais, CPMTC-IGC, Programa de Pós-Graduação em Geologia, Campus Pampulha, Belo Horizonte, $31270-901$ MG, Brazil \\ b Universidade Federal de Ouro Preto, DEGEO-EM, Campus Morro do Cruzeiro, Ouro Preto, 35400-000 MG, Brazil
}

\section{A R T I C L E I N F O}

\section{Article history:}

Received 14 May 2013

Received in revised form 16 November 2013

Accepted 24 November 2013

Available online 22 December 2013

Handling Editor: T. Gerya

\section{Keywords:}

Accretionary wedge

Suture

$\mathrm{U}-\mathrm{Pb}$ geochronology

Araçuaí orogen

Western Gondwana

\begin{abstract}
A B S T R A C T
The Araçuaí orogen represents a branch of the Brasiliano orogenic system developed between the São Francisco and Congo cratons in Neoproterozoic time. We conducted detailed studies on a complex schist belt located to the west of the Rio Doce magmatic arc, along the assumed suture zone of the Araçuaí orogen. This $30 \mathrm{~km}$-wide and $100 \mathrm{~km}$-long, NS-trending belt includes pelitic schists with intercalations of quartzites, metaultramafic schists and diopsidites, intruded by collisional granites. U-Pb ages from detrital zircon grains point to distinct provenances for different portions of the schist belt. The lower succession shows an age spectra and maximum depositional age (819 Ma) similar to passive margin deposits of the precursor basin. The upper succession yielded a maximum deposition age around $600 \mathrm{Ma}$, pointing toward the Rio Doce arc as the main sediment source. Thrust to the west onto the Guanhães basement and separated on the east from the Ediacaran Rio Doce magmatic arc by reverse-dextral faults, the schist belt exhibits the architecture of an asymmetric flower structure developed in transpressional regime. The distribution of metamorphic assemblages across the schist belt characterizes a collisional, Barrovian-type metamorphic zoning, in which the sillimanite, kyanite, staurolite and garnet zones are locally duplicated by thrusts. P-T conditions range from $700{ }^{\circ} \mathrm{C}$ at $7.5 \mathrm{kbar}$, at the western base of the pile, to $550{ }^{\circ} \mathrm{C}$ at $5.5 \mathrm{kbar}$, at the eastern top of the package. Zircon $\mathrm{U}-\mathrm{Pb}$ ages record metamorphic overprinting on the sheared top of the basement at $560 \pm 20$ Ma and crystallization of collisional granites at $544 \pm 10 \mathrm{Ma}$. Our results allow us to interpret the schist belt as a suture-related accretionary wedge and suggest that basin closure during the assembly of West Gondwanaland lasted to the Ediacaran-Cambrian boundary in the Araçuaí orogen.
\end{abstract}

(C) 2013 International Association for Gondwana Research. Published by Elsevier B.V. All rights reserved.

\section{Introduction}

A common challenge in Precambrian orogens is to reconstruct the pre-collisional palaeogeography and tectonic environments from basin fill to orogenic stages. In this regard, especially significant are sutures zones and associated rock assemblages that represent key elements to outline palaeoplate boundaries.

After three decades of systematic mapping and analytical studies, the geotectonic setting and major tectonic environments of the Araçuai orogen seems to be relatively well-established (Pedrosa-Soares et al., 2001; Alkmim et al., 2006; Pedrosa-Soares et al., 2007, 2008, 2011b). This orogen extends from the eastern border of the São Francisco craton to the Atlantic margin of southeastern Brazil (Fig. 1). Together with the West Congo belt of west-central Africa, the Araçuaí orogen forms a late Neoproterozoic-Cambrian orogenic edifice confined to

\footnotetext{
* Corresponding author. Tel.: +55 3197840412.

E-mail addresses: einpeixoto@gmail.com (E. Peixoto),pedrosa@pq.cnpq.br (A.C. Pedrosa-Soares), ffalkmim@gmail.com (F.F. Alkmim), ivodusin@yahoo.com.br (I.A. Dussin).

1 Research fellow of the Brazilian Scientific Council (CNPq).
}

an embayment surrounded by the São Francisco-Congo craton (Fig. 1). Such a peculiar setting and the paucity of data induced some former authors to portray the Araçuaí-West Congo orogen as a fully ensialic system (e.g., Trompette, 1994). The discovery of ophiolite slivers (Pedrosa-Soares et al., 1992, 1998) and the characterization of a magmatic arc in the Araçuaí orogen (Figueiredo and Campos Neto, 1993; Nalini et al., 2000; Pinto et al., 2000; Pedrosa-Soares et al., 2001; Martins et al., 2004; Vieira, 2007; Gonçalves et al., 2010; PedrosaSoares et al., 2011b) provided the basis to interpret the whole Araçuaí-West Congo system as an orogen generated after closure of a gulf partially floored by oceanic crust (Pedrosa-Soares et al., 2001, 2008). According to a model postulated by Alkmim et al. (2006), this precursor gulf, currently referred to as the Macaúbas basin, closed through a special mechanism called "nutcracker tectonics".

The western boundary of the magmatic arc preserved in the Araçuaí orogen, the so-called Rio Doce arc, is marked by the E-dipping Abre Campo shear zone (Cunningham et al., 1996, 1998; Peres et al., 2004). This shear zone juxtaposes the Rio Doce arc and its Palaeoproterozoic basement, on the east, with an Archaean-Palaeoproterozoic basement block and a schist belt, on the west (Fig. 2). Interpreted as a Neoproterozoic suture (Cunningham et al., 1996, 1998; Fischel, 1998; 

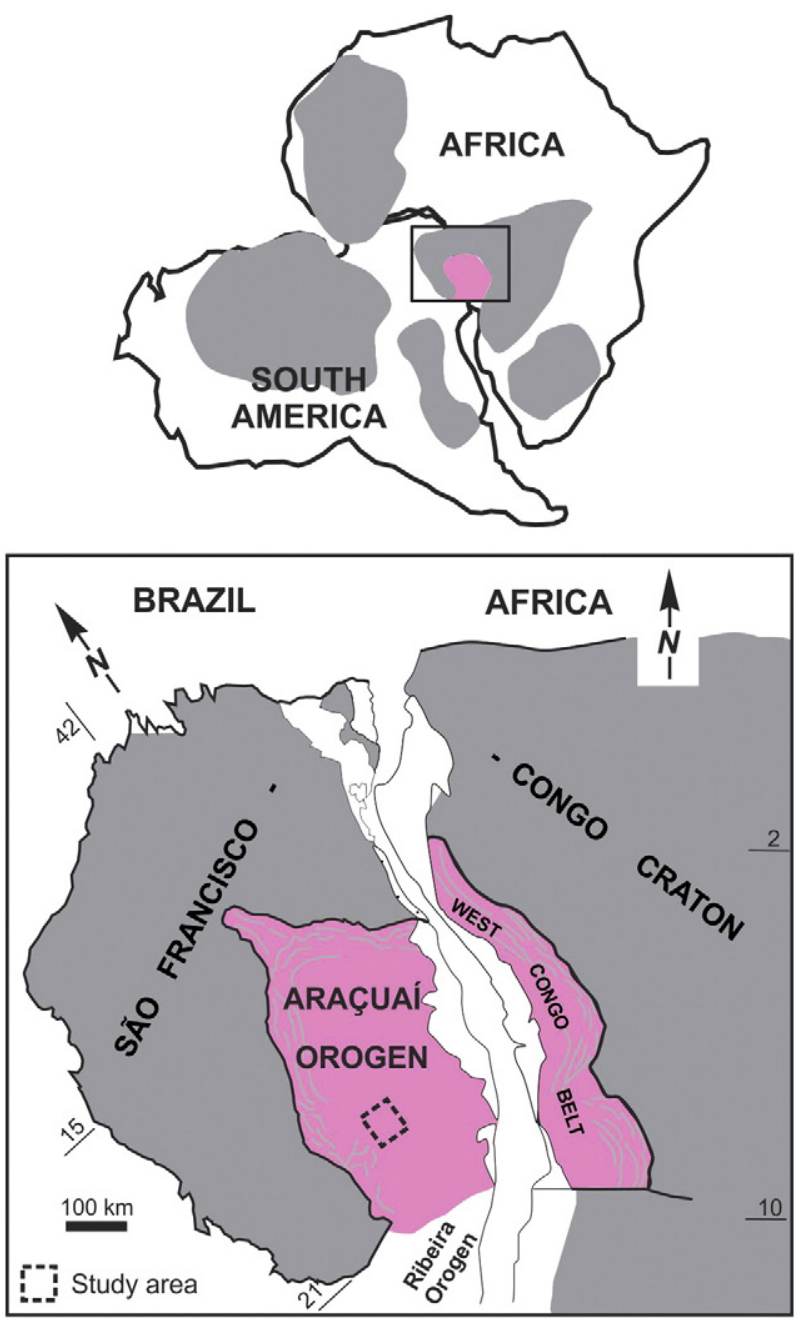

Fig. 1. Geotectonic setting of the Araçuaí-West Congo orogenic system and related cratons, with a box indicating the location of the focused region (modified from Alkmim et al., 2006).

Brueckner et al., 2000; Alkmim et al., 2006), the Abre Campo shear zone would thus represent an E-dipping subduction zone that promoted closure of the precursor Macaúbas basin, leading to the uplift of the Araçuaí orogen (Alkmim et al., 2006). If this interpretation is correct, the schist belt that occurs in the footwall of the Abre Campo shear zone would potentially correspond to an accretionary wedge. In order to test this hypothesis and contribute to a better reconstruction of the pre-collisional scenario of the Araçuaí-West Congo system, we performed a detailed investigation of a segment of the schist belt that follows the trace of the Abre Campo shear zone in the central portion of the Araçuaí orogen (Fig. 2). In this paper, we present the results of our study, which is based on detailed lithostructural mapping, quantitative thermobarometry, and zircon U-Pb (SHRIMP, LA-MC-ICP-MS) age determinations on metamorphosed sedimentary and igneous rocks.

\section{Geological setting}

The main rock units involved in the Araçuaí orogen comprise five lithotectonic assemblages, which represent: i) the basement assemblage; ii) the Tonian-Cryogenian Macaúbas basin succession and associated ophiolite bodies; iii) the Ediacaran Rio Doce magmatic arc and related sedimentary units; iv) orogenic (flysch-type) sedimentary deposits (Salinas Formation); and v) syn-to post-collisional magmatic suites (Fig. 2).
The basement assemblage includes Archaean-Palaeoproterozoic complexes as well as Palaeoproterozoic-Mesoproterozoic rift basins and associated igneous rocks (Dussin, 1994; Noce et al., 2007; Silva et al., 2011; Chemale et al., 2012a; Novo, 2013; Tedeschi, 2013).

The type-unit of the precursor basin of the Araçuaí orogen, the Macaúbas Group, records an evolution from continental rifting events and passive margin development to oceanic opening, in the time interval from the Tonian to Late Cryogenian (Pedrosa-Soares et al., 1998, 2011a; Uhlein et al., 1999; Pedrosa-Soares and Alkmim, 2011; Babinski et al., 2012). Composed of seven formations, the Macaúbas Group consists of an overall transgressive 1st order sequence that includes pre-rift, rift and passive margin to oceanic basin-fill units, partially influenced by a glacial event (Fig. 3). U-Pb data from detrital zircon grains point to a maximum depositional age of $900 \mathrm{Ma}$ for the proximal (western) Macaúbas Group (PedrosaSoares et al., 2000; Babinski et al., 2012).

The Ribeirão da Folha Formation, the youngest unit of the Macaúbas Group, consists of metamorphosed fine-grained turbidites, pelites, black-shales, cherts, banded iron formations and mafic volcanics that represent distal passive margin and ocean-floor deposits (Pedrosa-Soares et al., 1992, 1998; Queiroga et al., 2006; Queiroga, 2010; Pedrosa-Soares et al., 2011a). In the easternmost occurrences of the Ribeirão da Folha Formation, ocean-floor sediments are tectonically imbricated with slices of mafic and ultramafic rocks, representing dismembered ophiolite sections (Pedrosa-Soares et al., 1998; Aracema et al., 2000; Pedrosa-Soares et al., 2001, 2008). Zircons extracted from plagiogranite patches associated with an ophiolitic mafic section yielded magmatic $\mathrm{U}-\mathrm{Pb}$ ages around $650 \mathrm{Ma}$, constraining the minimum depositional age for the Ribeirão da Folha Formation and indicating that sea-floor spreading was still active in the Macaúbas basin until Late Cryogenian time (Queiroga et al., 2007; Queiroga, 2010).

An expanded calc-alkaline series composed of pre-collisional, I-type, plutons and volcanic rocks, whose ages fall in the interval from c. 630 to c. $580 \mathrm{Ma}$, occurs to the east of the Abre Campo shear zone and form the Rio Doce magmatic arc (e.g., Nalini et al., 2000; Pedrosa-Soares et al., 2001; Vieira, 2007; Gonçalves et al., 2010; Novo et al., 2010; PedrosaSoares et al., 2011b).

A large portion of the eastern sector of the Araçuaí orogen is underlain by the Nova Venécia Complex, which comprises paragneisses with intercalations of calc-silicate rocks (Pedrosa-Soares et al., 2008). Interpreted as back-arc basin-fill sediments, the Nova Venécia paragneisses contain detrital zircon grains dated at ca. $600 \mathrm{Ma}$ (Noce et al., 2004).

Separated from the older units by a regional unconformity, the Salinas Formation consists of a thick sequence of turbiditic wackes and pelites, containing lenses of conglomerates and calc-silicate rocks (Fig. 2). As indicated by provenance studies, the Salinas Formation, with a maximum depositional age of c. $588 \mathrm{Ma}$, filled the space between the approaching Rio Doce arc and the Macaúbas passive margin, as closure of the Araçuaí precursor basin reached an advanced stage in the time after $588 \mathrm{Ma}$ (Lima et al., 2002; Pedrosa-Soares et al., 2008; Santos et al., 2009).

The collisional stage is recorded in the Araçuaí orogen by a regional tectono-metamorphic event and production of an enormous volume of S-type granites mainly in the interval from $580 \mathrm{Ma}$ to $560 \mathrm{Ma}$, with peak around $575 \mathrm{Ma}$ (Fig. 2). Granite emplacement also took place during the gravity collapse of the orogen, which lasted from c. 535 Ma to c. $490 \mathrm{Ma}$ (De Campos et al., 2005; Marshak et al., 2006; Pedrosa-Soares et al., 2011b; Silva et al., 2011).

From a tectonic point of view, the Araçuaí orogen can be subdivided in two major domains: the low to medium grade fold-thrust belt bordering the São Francisco craton (named as Araçuaí belt by Almeida, 1977), and the internal high grade-granitic (crystalline) core of the orogen. Detailed descriptions of the overall structure of these domains can be found in Alkmim et al. (2006). 


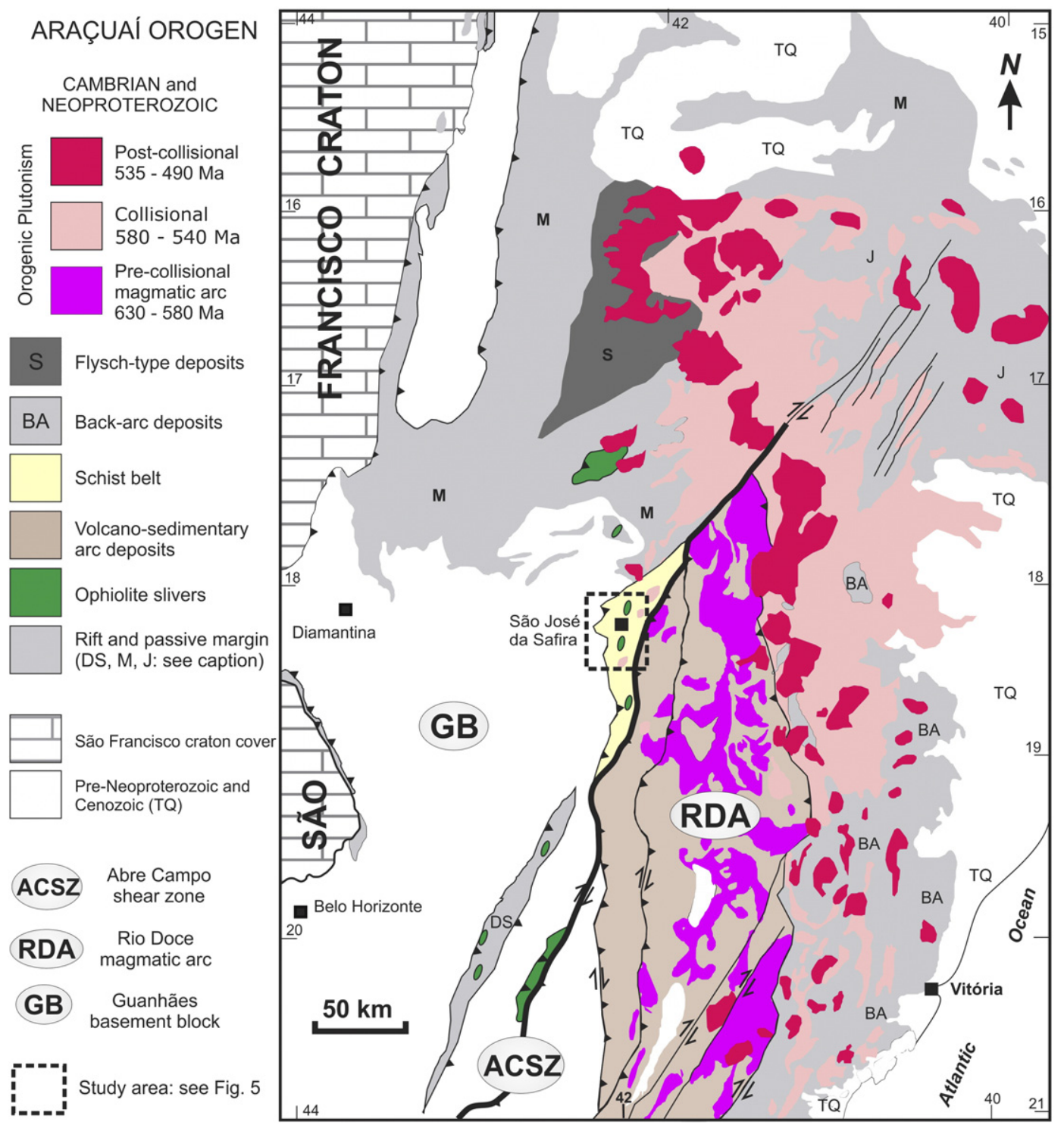

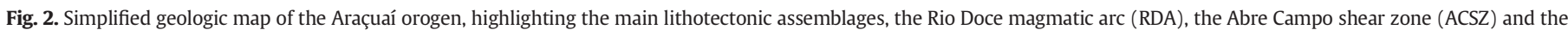
associated schist belt (modified from Pedrosa-Soares et al., 2008). M, Macaúbas Group; DS, Dom Silvério Group; J, Jequitinhonha Complex; S, Salinas Formation.

The external metamorphic fold-thrust belt extends from the cratonic edge to the eastern border of the Guanhães block (Fig. 2). W-verging thrusts and associated folds are the main regional fabric elements of its central sector, whereas NS to NE-trending dextral strike-slip shear zones dominate the structural picture of its southern segment.

The focus of this study is the roughly NS-trending schist belt that involves the Ribeirão da Folha and Salinas formations along the western edge of crystalline core of the orogen (Figs. 2 and 3). Around latitude $18^{\circ} \mathrm{S}$, this schist belt becomes narrower and continues further south following the trace of the Abre Campo shear zone, between the Guanhães basement block and the Rio Doce magmatic arc.

\section{The schist belt in the São José da Safira region}

The area we selected for our study is located in the vicinity of the town of São José da Safira (Minas Gerais State, southeastern Brazil). It encompasses a $36 \mathrm{~km}$-wide and $30 \mathrm{~km}$-long segment of the schist belt bounded to the east by the Abre Campo shear zone (Figs. 2 and 4). This particular portion of the schist belt was chosen for the quality of the exposures and nature of the rock units involved, i.e., distal passive margin sediments and ophiolite bodies, which potentially represent a suture-related accretionary wedge (Pedrosa-Soares et al.,
2001, 2008; Queiroga, 2010). In the next sections we present descriptions of the stratigraphy, structure and metamorphic zoning of the schist belt segment exposed near São José da Safira (Fig. 4).

\subsection{Stratigraphy}

The lithostratigraphic units involved in the schist belt in São José da Safira region are: the Archaean and Palaeoproterozoic basement gneisses, Neoproterozoic metasedimentary and metaultramafic rocks, the Brasilândia tonalite, and the Santa Rosa granite (Figs. 4 and 5).

The western portion of the study area exposes Archaean gneisses of the Guanhães block (Fig. 6). A shallow dipping thrust shear zone separates the basement gneisses from the Neoproterozoic schist package (Fig. 5). Basement gneisses also occur in the southeastern corner of the study area, where a reverse-dextral fault brought a slice of Palaeoproterozoic biotite-amphibole gneiss on top of the Neoproterozoic schists.

Our detailed mapping work allowed us to subdivide the package of Neoproterozoic metasedimenatry rocks into the informal units A and B (Figs. 4 and 5). The lower Unit A comprises an up to $2.5 \mathrm{~km}$ thick pile of coarse-grained, grey graphite-muscovite schist that grades upwards into biotite-muscovite schist containing lenses and layers of 


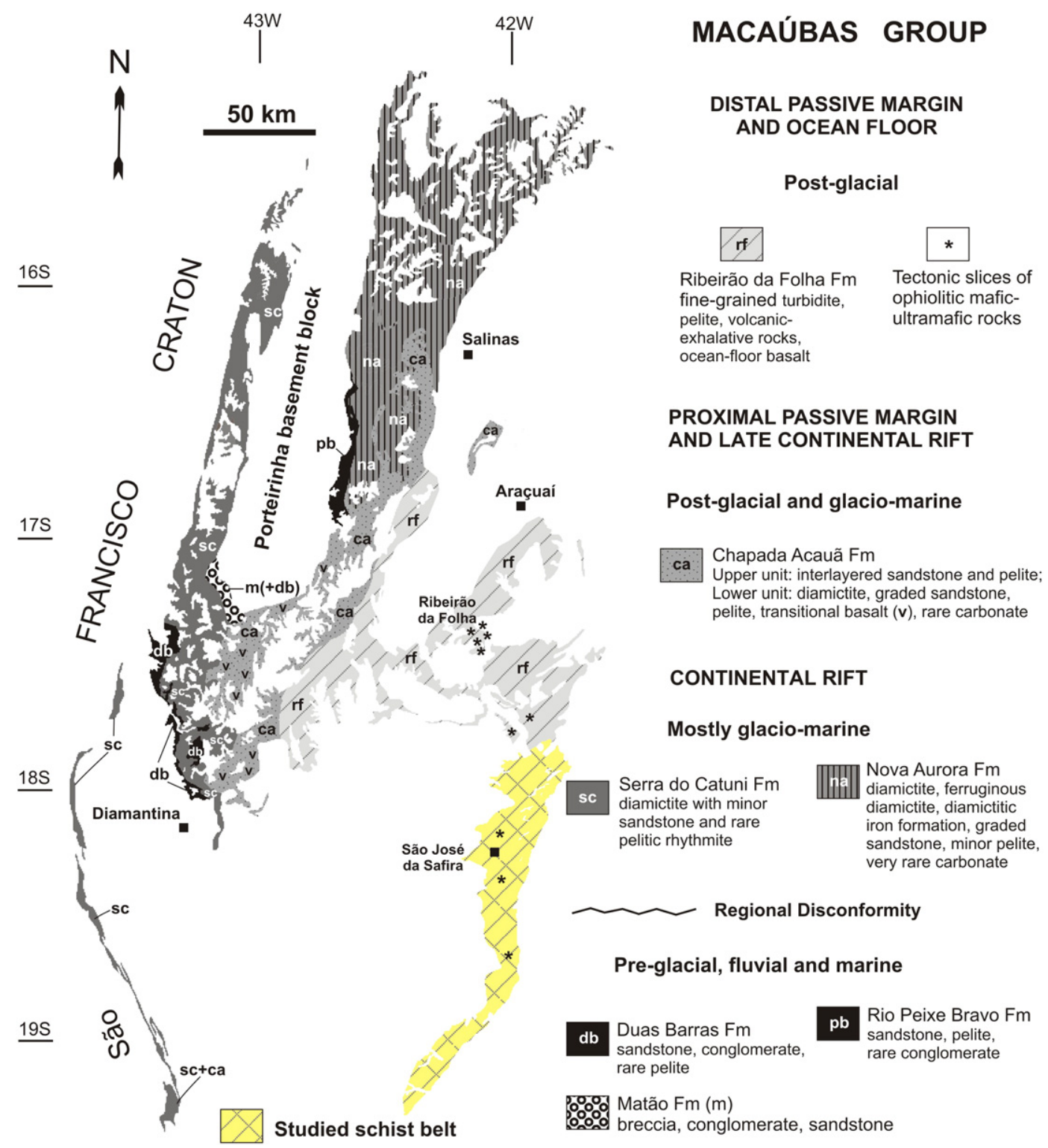

Fig. 3. Formations of the Macaúbas Group in the Araçuaí orogen (modified from Pedrosa-Soares et al., 2011a).

quartzite. Sillimanite and garnet are common accessory minerals in the pelitic schists (Fig. 6) exposed along a continuous layer in the hanging wall of the major thrust shear zone that marks the contact with the Guanhães basement block (Fig. 5). The dominant rock-types of the upper portion of Unit A are coarse-grained quartzites, occurring in thick layers and lenses intercalated with muscovite-rich quartzite, muscovite schist and garnet-muscovite-biotite schist. They contain spessartite, kyanite and staurolite as metamorphic-index minerals, and show lateral and vertical transitions to the rock-types of the lower portion of the Unit A. The main exposures of the upper portion of Unit $A$ are located in the northern half of the area, where two major thrusts caused its imbrication with Unit B (Figs. 4 and 5). Protoliths of the Unit A metasedimentary rocks are probably mud-rich pelites, carbonaceous pelites, and quartz-rich to clay-rich sandstones.

Cut by thrusts and dextral-reverse faults, Unit B occupies almost the entire eastern half of the mapped area (Fig. 4). Unit B comprises highly homogeneous light grey quartz-biotite schist with meta-arcose lenses in the lower portion, and a thick package of fine-grained biotite-quartz schists with intercalations of lenses and bands of calc-silicate rock in the upper part (Fig. 6). These schists also include plagioclase and muscovite, as well as garnet, staurolite, kyanite and sillimanite as metamorphic- index minerals, displaying a complex metamorphic zoning described in the forthcoming Section 3.4. The calc-silicate rock shows a diversified composition, including a fine-grained matrix composed of quartz, plagioclase, carbonate, biotite, muscovite, microcline, grossular, titanite and epidotes, as well as coarse-grained hornblende and diopside poikiloblasts. The whole package of Unit B probably derives from a wacke-pelite-carbonate succession of sedimentary protoliths.

Representing the western boundary of the Rio Doce magmatic arc, tectonic slices of a foliated tonalite are juxtaposed with Unit B rocks by a system of dextral-reverse faults (Figs. 4 and 5). The foliated Brasilândia tonalite, dated at c. $584 \mathrm{Ma}$, is rich in stretched mafic enclaves, and contains biotite, hornblende, epidote and clinozoisite (Tedeschi, 2013).

The $7 \mathrm{~km}$-long and $5 \mathrm{~km}$-wide Santa Rosa Granite intrudes the rocks of Unit B in the central portion of the area (Fig. 4). Previously interpreted as a post-collisional pluton (Pinto et al., 2000; Pedrosa-Soares et al., 2011b), this intrusion is made up of a foliated syenogranite that exhibits a relatively homogenous core and very heterogeneous margins, marked by a prominent foliation and numerous amphibolite enclaves (Fig. 6). Its mineral assemblage includes quartz (36-47\%), K-feldspar (43-45\%), plagioclase (5-15\%), biotite (3-10\%), and muscovite, garnet and zircon 


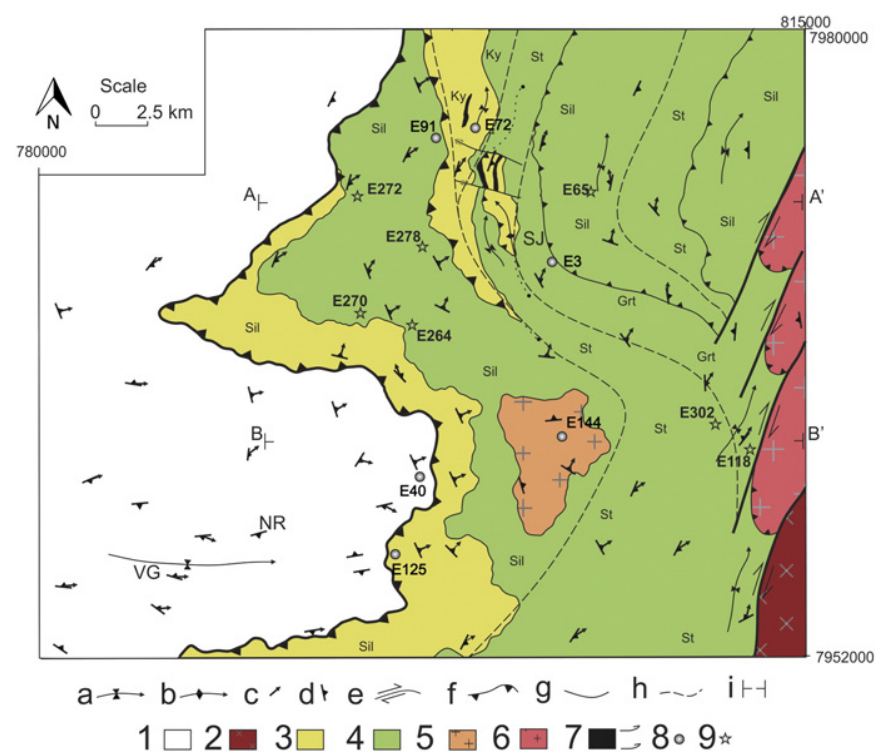

Fig. 4. Geologic map of the study area, São José da Safira region, eastern Minas Gerais State. 1, Archaean gneisses; 2, Palaeoproterozic gneisses; 3, Unit A (Ribeirão da Folha Formation); 4, Unit B (Salinas Formation); 5, Santa Rosa granite; 6, Brasilândia tonalite (Rio Doce arc); 7, metaultramafic bodies; 8 , samples for zircon U-Pb geochronological analysis; 9, samples used for geothermobarometric studies. a, synform; b, antiform; c, stretching lineation ( $\mathrm{Ln}$ ); d, regional foliation ( $\mathrm{Sn}$ ); e, strike-slip shear zones; f, thrust shear zones;

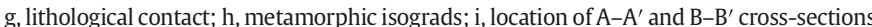
(see Fig. 5). Grt, garnet zone; St, staurolite zone; Ky, kyanite zone; Sil, sillimanite zone. Cities: VG, Virgolândia; NR, Nacip Raydan; SJ, São José da Safira.

as accessory minerals. Garnet becomes abundant along the border of the intrusion. With these characteristics the studied Santa Rosa intrusion can be more likely ascribed to the syn-collisional granites of the Araçuaí orogen.

\subsection{Tectonic slices of metaultramafic rocks}

Unit A hosts intercalations of remarkable lithotypes, represented by a variety of metaultramafic rocks, associated with thin lenses of banded iron formations (Neves and Ferreira, 1999; Aracema et al., 2000; Silva,
2000). Serpentinized olivine-orthopiroxene-bearing metaperidotite, ultramafic schists rich in anthophyllite, tremolite-actinolite and/or talc, and diopsidite form large pods and lenses within the upper metasedimentary package of Unit A (Fig. 6). Some of these bodies are large enough to show up in the local geological map (Fig. 4).

Bodies of serpentinized peridotite occur intercalated in quartzites of the upper portion of Unit A. They contain remnants of orthopyroxene and olivine replaced by serpentine with talc and carbonate. Chromite grains, dispersed in the rock or forming small aggregates, have been often observed in the peridotite. Anthophyllite-tremolite-talc schists are the main metaultramafic rocks found in the area, and seem to be metamorphic outcomes formed from peridotites (Fig. 6). Aracema et al. (2000) and Queiroga (2010) presented lithochemical and Sm$\mathrm{Nd}$ isotopic data that point to an ophiolite nature for these ultramafic rocks.

Diopsidite, a quite rare rock found in close association with ophiolites and suture zones (e.g., Pedrosa-Soares et al., 1998; Python et al., 2007; Santosh et al., 2010), occur at the base of a large metaultramafic lens exposed near São José da Safira (Fig. 6). This rock consists of decussate aggregates of up to $10 \mathrm{~cm}$ diopside megacrysts with interstitial milky quartz, cut by carbonate veins. Actinolite-tremolite partially replaces diopside in the contact zone between the diopsidite body and its host rock, which are in general ultramafic schists (Peixoto, 2013). Microprobe analysis of diopside crystals indicate high $\mathrm{Mg \#}\left(100 * \mathrm{Mg} / \mathrm{Mg}+\mathrm{Fe}_{\mathrm{t}}\right.$ ranging from $83 \%$ to $90 \%$ ) and high $\mathrm{Cr}_{2} \mathrm{O}_{3}$ values (0.06-0.84 wt.\%, on average $0.30 \mathrm{wt} . \%)$, but low contents of $\mathrm{Al}_{2} \mathrm{O}_{3}(0.04-0.21 \mathrm{wt} . \%$, on average 0.14 wt.\%), $\mathrm{Na}_{2} \mathrm{O}\left(0.09-0.27\right.$ wt.\%, on average 0,17 wt.\%) and $\mathrm{TiO}_{2}$ (0.00-0.12 wt.\%, on average 0.03$)$. This chemical composition suggests the action of hydrothermal fluids during the generation of the diopsidites (Python et al., 2007; Santosh et al., 2010).

\subsection{Structure and deformation history}

The geologic map and cross-sections (Figs. 4 and 5) show that the overall structure of the schist belt resembles an asymmetric, W-verging flower structure, in which the schist package, cut and bounded on both sides by oblique-slip faults, exhibits the shape of a wedge in 3D. The western boundary fault corresponds to a reverse-dextral thrust as attested by a variety of kinematic indicators. Showing a quite sinuous trace in map-view, the thrust surface is followed by an up to
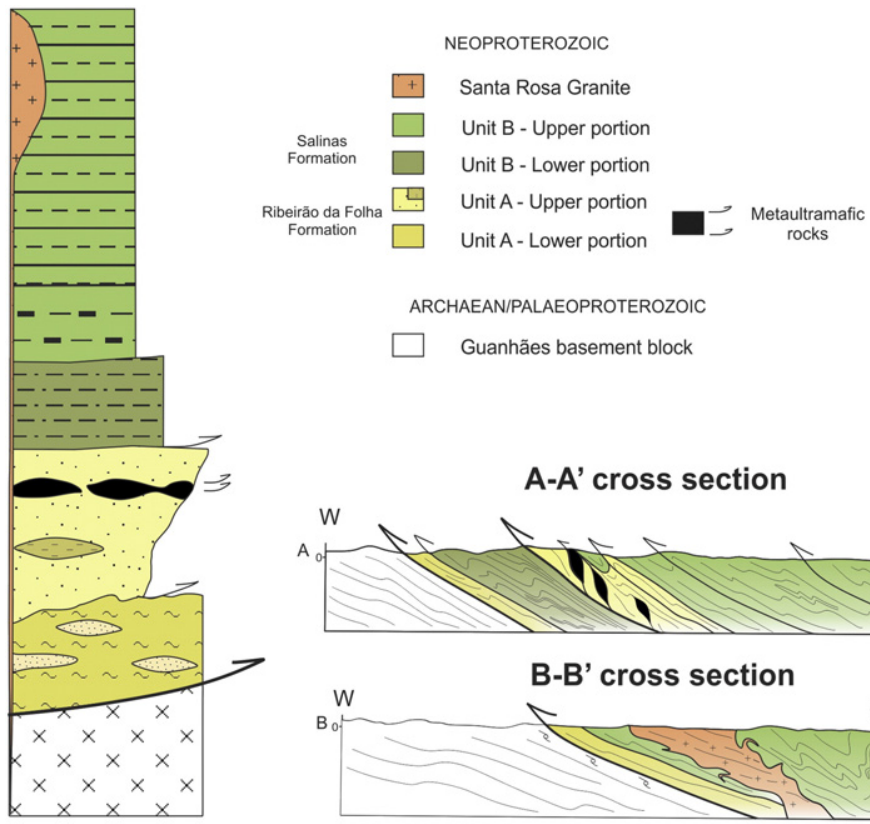

A-A' cross section
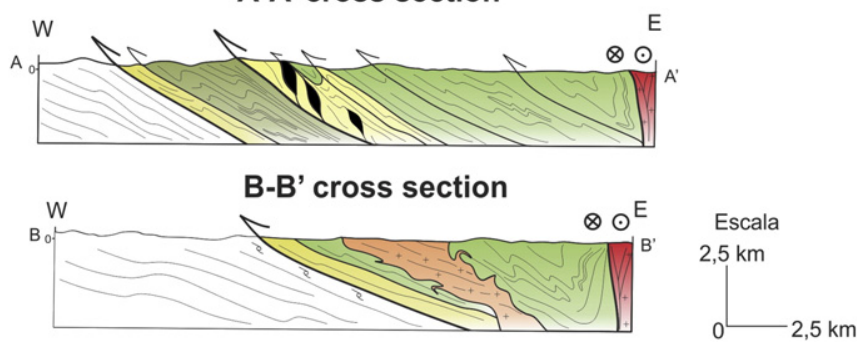

Fig. 5. Cross-sections and tectono-stratigraphic column of the schist belt in the São José da Safira area. 

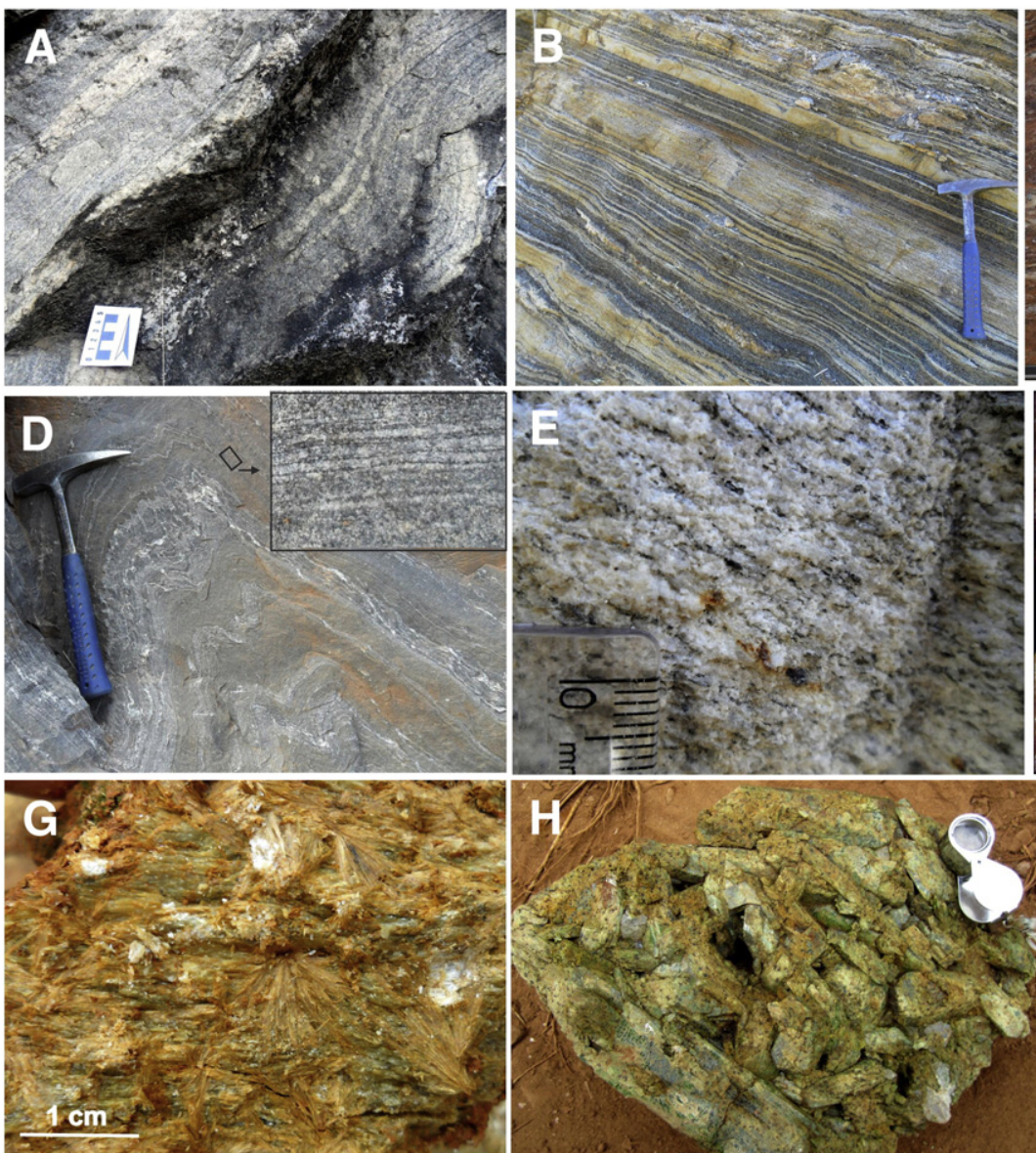
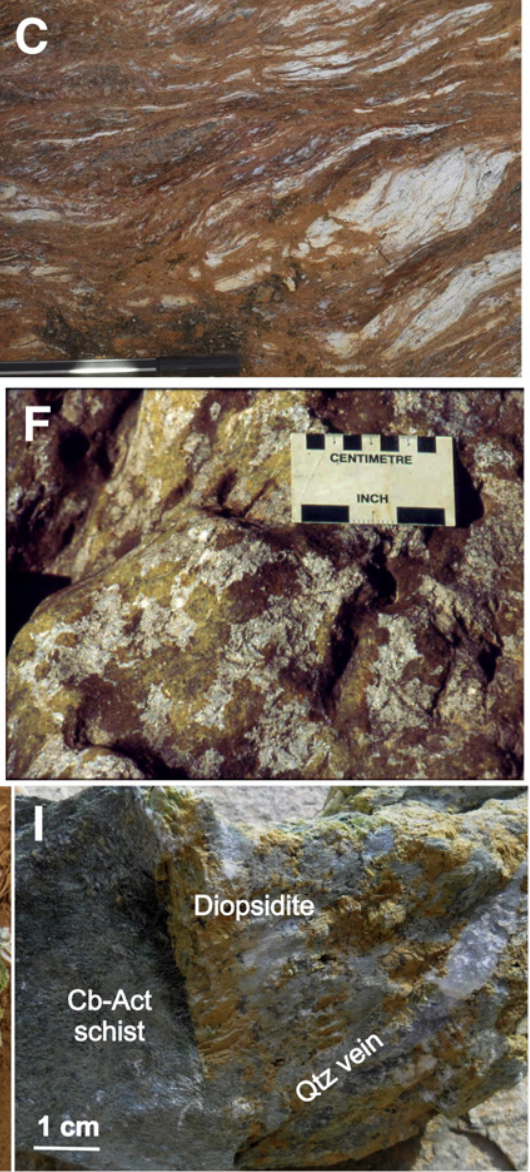

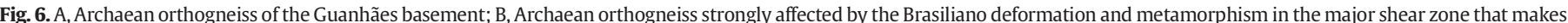

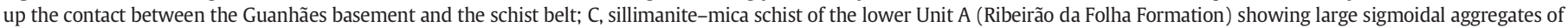

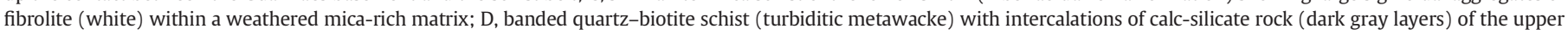

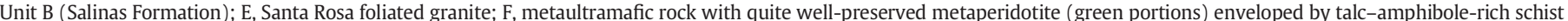

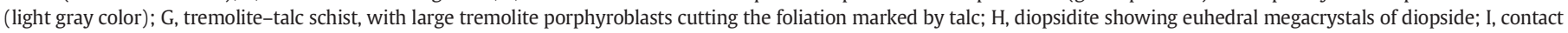
between carbonate $(\mathrm{Cb})$-actinolite(Act) schist and diopsidite, cut by a quartz(Qtz)-vein.

500 m-thick band of fine-laminated mylonites, developed in the footwall gneisses. The mylonitic foliation dips preferentially $20^{\circ}$ to $35^{\circ}$ ENE and the associated stretching lineation plunges $20^{\circ} \mathrm{NE}$. The eastern boundary of the flower structure is given by a set of five steeply dipping dextral-reverse to dextral strike-slip faults, also marked by mylonitic bands. Along these N20E-striking faults, the stretching lineation plunges $10^{\circ}$ to $25^{\circ} \mathrm{NNE}$. Various faults, ductile shear zones and associated folds affect the schists within the wedge. Their sinuous map-traces, following the trajectory of the western boundary thrust, define a culmination in the north and a recess in the south, where they seem to merge with the eastern boundary fault zone (Fig. 4).

The fabric elements documented in the basement rocks, schists and granitoids characterize two coaxial deformation phases, $D_{n}$ and $D_{n+1}$, which record the collisional event that led to the development of the Araçuaí orogen.

The dominant set of structures nucleated during the $\mathrm{D}_{\mathrm{n}}$ phase, which represents a general dextral transpression, generated under a NE-SW contractional field. Deformation during the $\mathrm{D}_{\mathrm{n}}$ phase intensively affected all units, so that primary or preexistent tectonic elements could not be documented in the study area. In the metasedimentary rocks, bedding is transposed into parallelism with the pervasive $S_{n}$ foliation. All contacts are sheared and not necessarily reflect the original depositional surfaces or older structures.

$F_{n}$ folds are tight to isoclinal and associated or truncated by ductile shear zones and faults. In the few exposures where they have been observed, the $\mathrm{B}_{\mathrm{n}}$ hinges plunge preferentially $15^{\circ}$ to $30^{\circ} \mathrm{NE}$ to NNE, exhibiting the same orientation of the $\mathrm{L}_{\mathrm{n}}$ stretching lineation. The NE to $E$-dipping $S_{n}$ foliation, representing the regional pervasive schistosity, occupies the axial plane of the $F_{n}$ folds and evolves to a mylonitic foliation $\left(\mathrm{S}_{\mathrm{mn}}\right)$ close to ductile shear zones and faults. Its orientation varies considerably along the study area, defining together with the fault traces the S-shaped structural grain of the schist belt (Figs. 4 and 7).
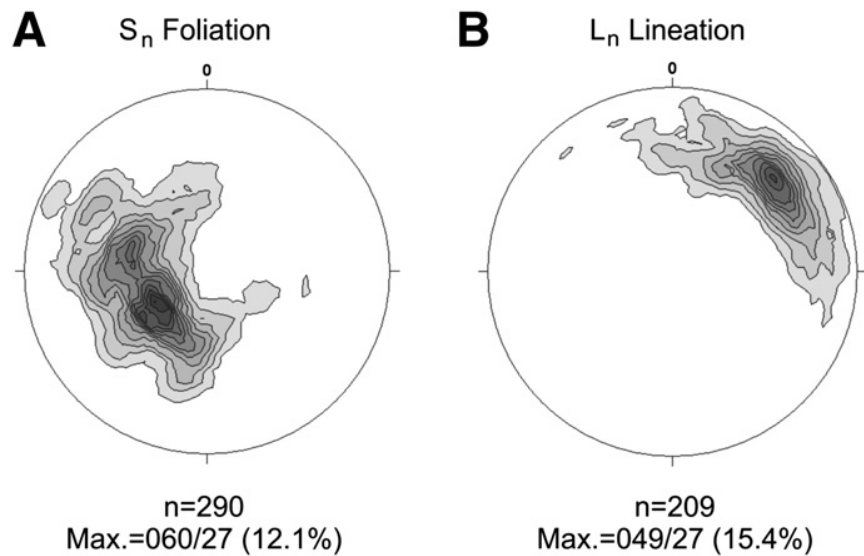

$\mathrm{n}=209$

Max. $=049 / 27(15.4 \%)$

Fig. 7. Stereonet plots of the small-scale structures related to $D_{n}$ and $D_{n+1}$ deformation phases: A, synoptic sterogram of contoured equal area plots to $S_{n}$ foliation; B, synoptic stereogram of contoured equal area plots to $L_{n}$ stretching lineation. 
Easily recognized on the $S_{n}$ foliation surface, the $L_{n}$ stretching lineation plunges NNE in the northeast and progressively changes its orientation, becoming ENE-plunging in the southwestern sector of the study area (Figs. 4 and 7). Furthermore, kinematic indicators of various categories (e.g., S-C structures, sigmoidal foliation trajectories, asymmetric porphyroclast tails, deformed veins and mineral aggregates) associated with the $\mathrm{L}_{\mathrm{n}}$-lineation attest a general SSW to SW-directed tectonic transport during the $\mathrm{D}_{\mathrm{n}}$ deformation phase.

The family of $D_{n+1}$ structures includes regional to outcrop-scale folds and an associated crenulation cleavage, which affect all previously described structures. The $F_{n+1}$ folds plunge preferentially ESE and are in general open, symmetric and upright. Locally, in areas close to the eastern boundary of the schist belt, the $F_{n+1}$ folds become tight, asymmetric and verge SW or NE. The associated $S_{n+1}$ crenulation cleavage strikes NW and dips preferentially NE.
Regional-scale $F_{n+1}$ folds seem to result from enhancement of WNW to E-W-trending basement highs and lows under the NE-SW contractional stress field. Thus, the curved map traces of the $D_{n}$ structures seem in part to derive from the interaction of the deformation front with basement highs and lows, and their subsequent enhancement during the late stage of the collisional deformation.

\subsection{Regional metamorphism}

The $D_{n}$ deformation phase was assisted by a regional metamorphic event under amphibolite facies conditions. The distribution of the mineral assemblages of pelitic schists characterizes a classical Barrovian metamorphic zoning, with higher grade mineral assemblages found on the west and lower grade on the east (Fig. 4). Thrusts and reverse faults duplicate the sillimanite zone in the northeastern sector of the
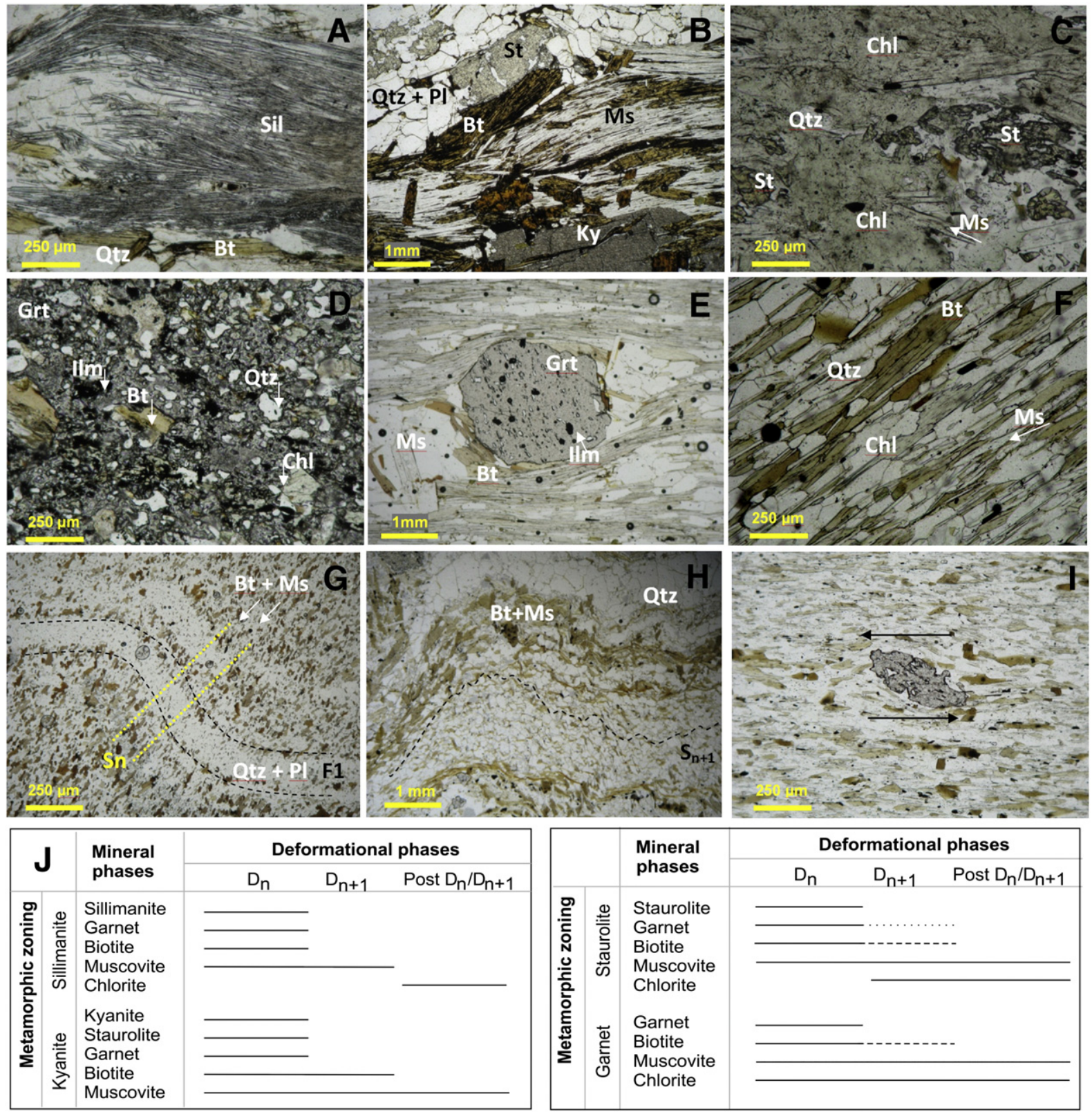

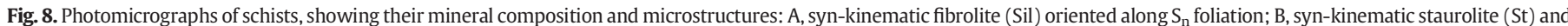

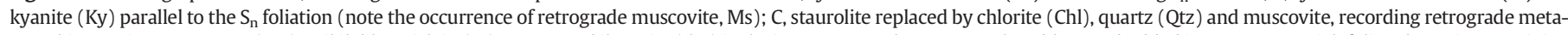

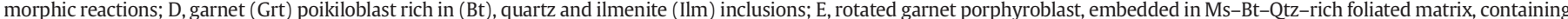

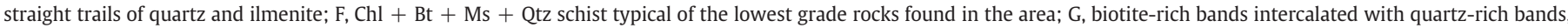

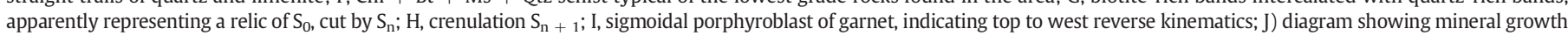
versus deformation (solid lines represent more frequent mineral phases). 
Table 1

Major element compositions and structural formula of representative mineral assemblages from metapelites and calc-silicate rocks.

\begin{tabular}{|c|c|c|c|c|c|c|c|c|c|c|c|c|c|c|c|c|c|c|c|c|}
\hline Sample & E65 & E65 & E65 & E65 & E65 & E118 & E118 & E118 & E118 & E118 & E118 & E264 & E264 & E264 & E264 & E264 & E264 & E270 & E270 & E270 \\
\hline \multirow[t]{3}{*}{ Min } & Grt & Grt & $\mathrm{Bt}$ & Pl & Ms & Grt & Pl & $\mathrm{Pl}$ & $\mathrm{Hbl}$ & $\mathrm{Hbl}$ & Ep & Grt & Grt & $\mathrm{Bt}$ & Pl & $\mathrm{Pl}$ & Ms & Grt & $\mathrm{Bt}$ & $\mathrm{Pl}$ \\
\hline & Near & Core & Near & Near & Near & Near & Near & Near & Near & Near & Near & Near & Core & Near & Near & Core & Near & Near & Near & Near \\
\hline & Rim & & Rim & Rim & Rim & Rim & Rim & Rim & Rim & Rim & Rim & Rim & & $\operatorname{Rim}$ & Rim & & Rim & Rim & Rim & Rim \\
\hline $\mathrm{SiO}_{2}$ & 39.00 & 38.68 & 36.43 & 66.79 & 49.59 & 39.36 & 45.51 & 61.02 & 50.80 & 49.13 & 39.63 & 37.61 & 37.11 & 35.52 & 64.21 & 64.50 & 47.46 & 39.28 & 36.57 & 62.36 \\
\hline $\mathrm{TiO}_{2}$ & 0.03 & 0.03 & 1.44 & 0.00 & 0.31 & 0.00 & 0.00 & 0.00 & 0.43 & 0.34 & 0.03 & 0.00 & 0.00 & 2.24 & 0.00 & 0.00 & 0.61 & 0.00 & 2.23 & 0.00 \\
\hline $\mathrm{Al}_{2} \mathrm{O}_{3}$ & 21.73 & 21.56 & 19.76 & 21.52 & 37.80 & 21.54 & 35.58 & 25.88 & 6.44 & 8.42 & 29.00 & 21.60 & 21.77 & 18.97 & 23.02 & 23.40 & 35.87 & 22.02 & 19.60 & 23.99 \\
\hline $\mathrm{Cr}_{2} \mathrm{O}_{3}$ & 0.00 & 0.00 & 0.00 & 0.00 & 0.00 & 0.00 & 0.00 & 0.00 & 0.00 & 0.00 & 0.00 & 0.00 & 0.00 & 0.00 & 0.00 & 0.00 & 0.00 & 0.00 & 0.00 & 0.00 \\
\hline $\mathrm{Fe}_{2} \mathrm{O}_{3}$ & 0.00 & 0.00 & 1.60 & 0.00 & 0.00 & 0.00 & 0.00 & 0.00 & 0.00 & 0.25 & 2.29 & 0.00 & 2.68 & 0.00 & 0.00 & 0.00 & 0.00 & 0.00 & 0.00 & 0.00 \\
\hline $\mathrm{FeO}$ & 33.61 & 32.52 & 18.20 & 0.00 & 0.97 & 17.02 & 0.00 & 0.00 & 13.90 & 14.40 & 3.15 & 31.24 & 30.65 & 19.39 & 0.00 & 0.00 & 1.02 & 31.36 & 19.44 & 0.00 \\
\hline $\mathrm{MnO}$ & 3.72 & 3.28 & 0.00 & 0.00 & 0.00 & 8.26 & 0.00 & 0.00 & 0.59 & 0.33 & 0.25 & 7.04 & 5.71 & 0.00 & 0.00 & 0.00 & 0.00 & 2.59 & 0.00 & 0.00 \\
\hline MgO & 3.36 & 3.45 & 10.26 & 0.00 & 0.49 & 1.20 & 0.00 & 0.00 & 12.93 & 11.96 & 0.00 & 2.55 & 3.05 & 9.44 & 0.00 & 0.00 & 0.64 & 3.29 & 9.51 & 0.00 \\
\hline $\mathrm{CaO}$ & 1.26 & 1.52 & 0.02 & 2.58 & 0.00 & 14.38 & 20.16 & 7.80 & 12.87 & 12.71 & 25.03 & 1.55 & 1.81 & 0.00 & 4.40 & 4.57 & 0.00 & 1.90 & 0.00 & 5.88 \\
\hline $\mathrm{Na}_{2} \mathrm{O}$ & 0.08 & 0.04 & 0.28 & 9.21 & 1.76 & 0.00 & 0.51 & 6.48 & 0.48 & 0.56 & 0.00 & 0.00 & 0.04 & 0.16 & 7.91 & 8.11 & 0.86 & 0.00 & 0.16 & 7.19 \\
\hline $\mathrm{K}_{2} \mathrm{O}$ & 0.00 & 0.00 & 7.71 & 0.05 & 7.79 & 0.00 & 0.00 & 0.00 & 0.38 & 0.41 & 0.00 & 0.00 & 0.00 & 8.29 & 0.10 & 0.10 & 9.90 & 0.00 & 8.99 & 0.10 \\
\hline Totals & 102.79 & 101.08 & 95.70 & 100.15 & 98.71 & 101.76 & 101.76 & 101.18 & 98.82 & 98.52 & 99.38 & 101.59 & 102.82 & 94.01 & 99.64 & 100.68 & 96.36 & 100.44 & 96.50 & 99.52 \\
\hline Oxygens & 12.0 & 12.0 & 11.0 & 8.0 & 11.0 & 12.0 & 8.0 & 8.0 & 23.0 & 23.0 & 12.5 & 12.0 & 12.0 & 11.0 & 8.0 & 8.0 & 11.0 & 12.0 & 11.0 & 8.0 \\
\hline $\mathrm{Si}$ & 3.036 & 3.047 & 2.717 & 2.914 & 3.122 & 3.043 & 2.068 & 2.676 & 7.324 & 7.128 & 3.042 & 2.989 & 2.916 & 2.719 & 2.829 & 2.817 & 3.103 & 3.084 & 2.728 & 2.764 \\
\hline $\mathrm{Ti}$ & 0.002 & 0.002 & 0.081 & 0.000 & 0.015 & 0.000 & 0.000 & 0.000 & 0.047 & 0.037 & 0.002 & 0.000 & 0.000 & 0.129 & 0.000 & 0.000 & 0.030 & 0.000 & 0.125 & 0.000 \\
\hline $\mathrm{Al}$ & 1.994 & 2.002 & 1.737 & 1.107 & 2.806 & 1.963 & 1.906 & 1.338 & 1.095 & 1.440 & 2.624 & 2.024 & 2.016 & 1.712 & 1.196 & 1.205 & 2.765 & 2.038 & 1.724 & 1.254 \\
\hline $\mathrm{Cr}$ & 0.000 & 0.000 & 0.000 & 0.000 & 0.000 & 0.000 & 0.000 & 0.000 & 0.000 & 0.000 & 0.000 & 0.000 & 0.000 & 0.000 & 0.000 & 0.000 & 0.000 & 0.000 & 0.000 & 0.000 \\
\hline $\mathrm{Fe}^{3}$ & 0.000 & 0.000 & 0.090 & 0.000 & 0.000 & 0.000 & 0.000 & 0.000 & 0.000 & 0.028 & 0.137 & 0.000 & 0.158 & 0.000 & 0.000 & 0.000 & 0.000 & 0.000 & 0.000 & 0.000 \\
\hline $\mathrm{Fe}^{2}$ & 2.188 & 2.143 & 1.134 & 0.000 & 0.051 & 1.100 & 0.000 & 0.000 & 1.676 & 1.748 & 0.198 & 2.077 & 2.014 & 1.241 & 0.000 & 0.000 & 0.056 & 2.059 & 1.213 & 0.000 \\
\hline $\mathrm{Mn}$ & 0.245 & 0.219 & 0.000 & 0.000 & 0.000 & 0.541 & 0.000 & 0.000 & 0.072 & 0.041 & 0.016 & 0.474 & 0.380 & 0.000 & 0.000 & 0.000 & 0.000 & 0.172 & 0.000 & 0.000 \\
\hline $\mathrm{Mg}$ & 0.390 & 0.405 & 1.140 & 0.000 & 0.046 & 0.138 & 0.000 & 0.000 & 2.778 & 2.586 & 0.000 & 0.302 & 0.357 & 1.077 & 0.000 & 0.000 & 0.062 & 0.385 & 1.057 & 0.000 \\
\hline $\mathrm{Ca}$ & 0.105 & 0.128 & 0.002 & 0.121 & 0.000 & 1.191 & 0.982 & 0.366 & 1.988 & 1.976 & 2.058 & 0.132 & 0.152 & 0.000 & 0.208 & 0.214 & 0.000 & 0.160 & 0.000 & 0.279 \\
\hline $\mathrm{Na}$ & 0.012 & 0.006 & 0.040 & 0.779 & 0.215 & 0.000 & 0.045 & 0.551 & 0.134 & 0.158 & 0.000 & 0.000 & 0.006 & 0.024 & 0.676 & 0.687 & 0.109 & 0.000 & 0.023 & 0.618 \\
\hline K & 0.000 & 0.000 & 0.734 & 0.003 & 0.626 & 0.000 & 0.000 & 0.000 & 0.070 & 0.076 & 0.000 & 0.000 & 0.000 & 0.810 & 0.006 & 0.006 & 0.826 & 0.000 & 0.855 & 0.006 \\
\hline Sum & 7.972 & 7.953 & 7.676 & 4.923 & 6.881 & 7.976 & 5.001 & 4.931 & 15.184 & 15.226 & 8.078 & 7.998 & 8.000 & 7.713 & 4.914 & 4.927 & 6.952 & 7.897 & 7.725 & 4.921 \\
\hline
\end{tabular}

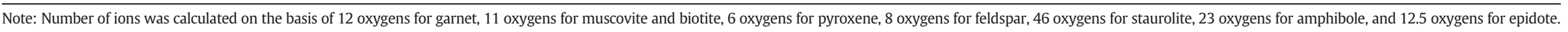




\begin{tabular}{|c|c|c|c|c|c|c|c|c|c|c|c|c|c|c|c|c|c|c|c|c|c|}
\hline E270 & E272 & E272 & E272 & E272 & E272 & E272 & E272 & E272 & E278 & E278 & E278 & E278 & E278 & E302 & E302 & E302 & E302 & E302 & E302 & E302 & E302 \\
\hline $\mathrm{Ilm}$ & Grt & Grt & $\mathrm{Bt}$ & $\mathrm{Bt}$ & $\mathrm{Pl}$ & $\mathrm{Pl}$ & Ms & Ms & Grt & Grt & $\mathrm{Bt}$ & $\mathrm{Pl}$ & Ms & Grt & Grt & Grt & $\mathrm{Bt}$ & $\mathrm{Pl}$ & Ms & St & St \\
\hline Near & Near & Core & Near & Core & Near & Centro & Near & Core & Near & Core & Near & Near & Near & Near & Core & Core & Near & Near & Near & Near & Core \\
\hline Rim & Rim & & Rim & & Rim & & Rim & & Rim & & Rim & Rim & Rim & Rim & & & Rim & Rim & Rim & Rim & \\
\hline 0.00 & 37.16 & 38.02 & 36.91 & 36.43 & 64.20 & 63.97 & 47.18 & 46.31 & 38.96 & 39.05 & 36.85 & 65.73 & 49.55 & 38.16 & 37.86 & 37.81 & 36.30 & 68.01 & 48.26 & 27.90 & 28.76 \\
\hline 54.00 & 0.00 & 0.00 & 2.68 & 2.76 & 0.00 & 0.00 & 0.80 & 0.83 & 0.00 & 0.00 & 2.57 & 0.00 & 0.64 & 0.00 & 0.01 & 0.02 & 1.58 & 0.00 & 0.26 & 0.51 & 0.61 \\
\hline 0.00 & 21.51 & 21.66 & 19.26 & 19.24 & 23.48 & 23.18 & 34.30 & 34.78 & 21.94 & 21.81 & 19.42 & 21.64 & 37.46 & 21.53 & 21.49 & 20.95 & 19.21 & 21.50 & 36.89 & 53.50 & 54.39 \\
\hline 0.00 & 0.00 & 0.00 & 0.00 & 0.00 & 0.00 & 0.00 & 0.00 & 0.00 & 0.00 & 0.00 & 0.00 & 0.00 & 0.00 & 0.00 & 0.00 & 0.00 & 0.00 & 0.00 & 0.00 & 0.00 & 0.00 \\
\hline 0.00 & 1.86 & 0.78 & 0.00 & 0.00 & 0.00 & 0.00 & 0.00 & 0.00 & 0.00 & 0.00 & 0.00 & 0.00 & 1.42 & 0.00 & 0.00 & 0.00 & 0.00 & 0.00 & 0.00 & 0.00 & 0.00 \\
\hline 44.96 & 28.99 & 26.55 & 19.52 & 19.90 & 0.00 & 0.00 & 1.35 & 1.25 & 31.45 & 31.22 & 18.31 & 0.00 & 0.55 & 33.94 & 33.33 & 28.72 & 18.18 & 0.00 & 0.91 & 13.16 & 13.59 \\
\hline 1.07 & 7.21 & 8.89 & 0.23 & 0.30 & 0.00 & 0.00 & 0.00 & 0.00 & 5.97 & 5.17 & 0.22 & 0.00 & 0.00 & 4.37 & 4.23 & 8.29 & 0.00 & 0.00 & 0.00 & 0.29 & 0.25 \\
\hline 0.52 & 2.68 & 1.72 & 9.04 & 9.00 & 0.00 & 0.00 & 0.82 & 0.73 & 3.29 & 3.43 & 10.10 & 0.00 & 0.51 & 3.07 & 3.01 & 2.76 & 10.47 & 0.00 & 0.40 & 1.74 & 1.89 \\
\hline 0.00 & 2.62 & 5.27 & 0.00 & 0.00 & 5.24 & 5.21 & 0.00 & 0.04 & 0.89 & 1.12 & 0.00 & 2.62 & 0.00 & 1.18 & 1.04 & 0.53 & 0.00 & 2.37 & 0.00 & 0.00 & 0.01 \\
\hline 0.00 & 0.00 & 0.00 & 0.13 & 0.16 & 7.78 & 7.63 & 0.66 & 0.75 & 0.00 & 0.03 & 0.31 & 9.06 & 1.07 & 0.00 & 0.00 & 0.00 & 0.22 & 9.12 & 1.50 & 0.00 & 0.03 \\
\hline 0.00 & 0.00 & 0.03 & 9.04 & 9.01 & 0.20 & 0.20 & 10.32 & 10.36 & 0.00 & 0.02 & 8.41 & 0.00 & 7.73 & 0.00 & 0.00 & 0.00 & 8.08 & 0.00 & 8.38 & 0.00 & 0.00 \\
\hline 100.55 & 102.04 & 102.92 & 96.81 & 96.80 & 100.90 & 100.19 & 95.43 & 95.05 & 102.50 & 101.85 & 96.19 & 99.05 & 98.93 & 102.25 & 100.97 & 99.08 & 94.04 & 101.00 & 96.60 & 97.10 & 99.53 \\
\hline 3.0 & 12.0 & 12.0 & 11.0 & 11.0 & 8.0 & 8.0 & 11.0 & 11.0 & 12.0 & 12.0 & 11.0 & 8.0 & 11.0 & 12.0 & 12.0 & 12.0 & 11.0 & 8.0 & 11.0 & 46.0 & 46.0 \\
\hline 0.000 & 2.941 & 2.978 & 2.747 & 2.721 & 2.803 & 2.811 & 3.130 & 3.089 & 3.037 & 3.053 & 2.737 & 2.900 & 3.113 & 3.006 & 3.013 & 3.057 & 2.751 & 2.934 & 3.117 & 7.777 & 7.826 \\
\hline 1.011 & 0.000 & 0.000 & 0.150 & 0.155 & 0.000 & 0.000 & 0.040 & 0.042 & 0.000 & 0.000 & 0.144 & 0.000 & 0.030 & 0.000 & 0.001 & 0.001 & 0.090 & 0.000 & 0.013 & 0.107 & 0.125 \\
\hline 0.000 & 2.007 & 2.000 & 1.690 & 1.694 & 1.209 & 1.201 & 2.683 & 2.735 & 2.016 & 2.010 & 1.700 & 1.126 & 2.774 & 2.000 & 2.016 & 1.997 & 1.717 & 1.094 & 2.809 & 17.582 & 17.448 \\
\hline 0.000 & 0.000 & 0.000 & 0.000 & 0.000 & 0.000 & 0.000 & 0.000 & 0.000 & 0.000 & 0.000 & 0.000 & 0.000 & 0.000 & 0.000 & 0.000 & 0.000 & 0.000 & 0.000 & 0.000 & 0.000 & 0.000 \\
\hline 0.000 & 0.111 & 0.046 & 0.000 & 0.000 & 0.000 & 0.000 & 0.000 & 0.000 & 0.000 & 0.000 & 0.000 & 0.000 & 0.067 & 0.000 & 0.000 & 0.000 & 0.000 & 0.000 & 0.000 & 0.000 & 0.000 \\
\hline 0.936 & 1.919 & 1.739 & 1.215 & 1.243 & 0.000 & 0.000 & 0.075 & 0.070 & 2.050 & 2.041 & 1.137 & 0.000 & 0.029 & 2.236 & 2.218 & 1.942 & 1.152 & 0.000 & 0.049 & 3.068 & 3.093 \\
\hline 0.023 & 0.483 & 0.590 & 0.015 & 0.019 & 0.000 & 0.000 & 0.000 & 0.000 & 0.394 & 0.342 & 0.014 & 0.000 & 0.000 & 0.292 & 0.285 & 0.568 & 0.000 & 0.000 & 0.000 & 0.068 & 0.058 \\
\hline 0.019 & 0.316 & 0.201 & 1.003 & 1.002 & 0.000 & 0.000 & 0.081 & 0.073 & 0.382 & 0.400 & 1.118 & 0.000 & 0.048 & 0.360 & 0.357 & 0.333 & 1.183 & 0.000 & 0.039 & 0.723 & 0.766 \\
\hline 0.000 & 0.222 & 0.442 & 0.000 & 0.000 & 0.245 & 0.245 & 0.000 & 0.003 & 0.074 & 0.094 & 0.000 & 0.124 & 0.000 & 0.100 & 0.089 & 0.046 & 0.000 & 0.110 & 0.000 & 0.000 & 0.003 \\
\hline 0.000 & 0.000 & 0.000 & 0.019 & 0.023 & 0.659 & 0.650 & 0.085 & 0.097 & 0.000 & 0.005 & 0.045 & 0.775 & 0.130 & 0.000 & 0.000 & 0.000 & 0.032 & 0.763 & 0.188 & 0.000 & 0.016 \\
\hline 0.000 & 0.000 & 0.003 & 0.858 & 0.859 & 0.011 & 0.011 & 0.874 & 0.882 & 0.000 & 0.002 & 0.797 & 0.000 & 0.620 & 0.000 & 0.000 & 0.000 & 0.781 & 0.000 & 0.691 & 0.000 & 0.000 \\
\hline 1.989 & 8.000 & 8.000 & 7.697 & 7.718 & 4.927 & 4.919 & 6.968 & 6.991 & 7.955 & 7.946 & 7.691 & 4.925 & 6.812 & 7.994 & 7.979 & 7.943 & 7.708 & 4.901 & 6.905 & 29.325 & 29.334 \\
\hline
\end{tabular}


Table 2

Geothermobarometric data for pelitic schists.

\begin{tabular}{|c|c|c|c|c|c|c|c|}
\hline \multirow[t]{3}{*}{ Sample } & \multirow[t]{3}{*}{ Zone } & \multirow[t]{3}{*}{ Mineral assemblages } & \multirow{2}{*}{\multicolumn{2}{|c|}{ THERMOCALC }} & \multicolumn{3}{|c|}{ Conventional thermometry } \\
\hline & & & & & \multirow{2}{*}{$\frac{\mathrm{T} 76}{\mathrm{~T}\left({ }^{\circ} \mathrm{C}\right)}$} & \multirow{2}{*}{$\frac{\mathrm{FS} 78}{\mathrm{~T}\left({ }^{\circ} \mathrm{C}\right)}$} & \multirow{2}{*}{$\frac{\text { HL77 }}{\mathrm{T}\left({ }^{\circ} \mathrm{C}\right)}$} \\
\hline & & & $\mathrm{T}\left({ }^{\circ} \mathrm{C}\right)$ & $\mathrm{P}$ (kbar) & & & \\
\hline E118 & Grt & $\mathrm{Hbl}+\mathrm{Pl}+\mathrm{Czo}+\mathrm{Ep}+\mathrm{Qtz}+\mathrm{Ab}+\mathrm{An}+\mathrm{Ttn}+\mathrm{Py}$ & $553 \pm 47$ & $5.5 \pm 1.1$ & - & - & - \\
\hline E302 & St & $\mathrm{St}+\mathrm{Grt}+\mathrm{Pl}+\mathrm{Bt}+\mathrm{Ms}+\mathrm{Qtz}$ & $705 \pm 36$ & $7.6 \pm 1.6$ & 578 & 545 & 558 \\
\hline E264 & Sil & $\mathrm{Grt}+\mathrm{Pl}+\mathrm{Bt}+\mathrm{Ms}+\mathrm{Qtz}$ & $657 \pm 22$ & $6.2 \pm 0.8$ & 595 & 566 & 572 \\
\hline E270 & Sil & $\mathrm{Grt}+\mathrm{Pl}+\mathrm{Bt}+\mathrm{Qtz}$ & - & - & 664 & 656 & 632 \\
\hline E272 & Sil & $\mathrm{Grt}+\mathrm{Pl}+\mathrm{Bt}+\mathrm{Ms}+\mathrm{Qtz}$ & $671 \pm 30$ & $7.8 \pm 1.0$ & 626 & 607 & 600 \\
\hline E278 & Sil & $\mathrm{Sil}+\mathrm{Grt}+\mathrm{Pl}+\mathrm{Bt}+\mathrm{Ms}+\mathrm{Qtz}$ & - & - & 628 & 609 & 601 \\
\hline E65 & Sil & $\mathrm{Grt}+\mathrm{Pl}+\mathrm{Bt}+\mathrm{Ms}+\mathrm{Qtz}$ & $694 \pm 27$ & $7.5 \pm 1.2$ & 630 & 611 & 602 \\
\hline
\end{tabular}

schist belt, indicating that deformation was still active after ceasing the progressive metamorphic regime.

All index minerals, like garnet, staurolite, kyanite and sillimanite mark the $S_{n}$ regional schistosity and $L_{n}$-lineation, being thus synkinematic in respect to the main collisional deformation. Garnet also shows textural relationships typical of pre-, syn- and post-kinematic growth. Its inclusions, consisting of quartz, ilmenite, chlorite, muscovite and biotite, are decussate or form plane-parallel trails, possibly recording greenschist facies conditions of the progressive metamorphism. The microstructures exhibited by the pelitic schists involve the Barroviantype mineral assemblages typical of a regional progressive metamorphism. These minerals are in turn affected by retrogression reactions (Fig. 8).

The $S_{n+1}$ crenulation domains are marked by muscovite, chlorite and biotite. Staurolite is frequently folded around the crenulation domains and retro-metamorphosed to muscovite and chlorite. The latter minerals also occur as post-kinematic porphyroblasts in respect to the $D_{n+1}$ fabric elements. Replacement of biotite and garnet by chlorite is also a common reaction in the studied rocks and reflects a widespread retrogressive metamorphism.

\section{Geothermobarometry}

Owing to the strong chemical weathering common in the region, adequate samples to be used for mineral chemistry analysis are difficult to find. Thus, only seven samples (six pelitic schists and one calc-silicate rock), all of them from the Unit B, were analyzed for quantitative geothermobarometric evaluations. These samples are representative of the garnet (E118), staurolite (E302) and sillimanite (E264, E270, E272, E278 and E65) zones of the regional metamorphism (Fig. 4).

\subsection{Analytical procedures}

Mineral compositions were analyzed on a JOEL JXA-8900RL electron microprobe of the Microanalysis Laboratory of the Federal University of Minas Gerais, Brazil. The operating conditions were: $15 \mathrm{kV}$ acceleration voltages, $5 \mu \mathrm{m}$ beam diameter and $20 \mathrm{nA}$ beam current. Wider beam diameter was used for plagioclase. The elements analyzed were $\mathrm{Si}, \mathrm{Ti}$, $\mathrm{Al}, \mathrm{Fe}, \mathrm{Mn}, \mathrm{Mg}, \mathrm{Ca}, \mathrm{Na}$ and $\mathrm{K}$, together with natural (quartz, rutile, magnetite, rodonite, jadeite and microcline) and synthetic (alumina, magnesia and anorthite) standards.

Analyses were performed on garnet, biotite, muscovite, plagioclase, staurolite and ilmenite, in pelitic schists, and on amphibole, plagioclase, garnet, epidote, titanite and ilmenite, in calc-silicate rocks. In order to check the chemical homogeneity, the analyses were run at least on three distinct fields for each thin section, avoiding external borders. Each crystal was analyzed at least at three points, one in the core and two at borders, in sites where the mineral assemblage is in apparent equilibrium. In order to recognize chemical zoning, cross-sections through 13 to 15 spots were performed in garnet porphyroblasts. Table 1 presents the most representative mineral chemistry data.

Temperature and pressure values were calculated using avPT (average P-T; Powell and Holland, 2008), an optimized method of THERMOCALC 3.2 (Powell et al., 1998), and conventional thermometry with calibrations by Thompson (1976), Holdoway and Lee (1977) and Ferry and Spear
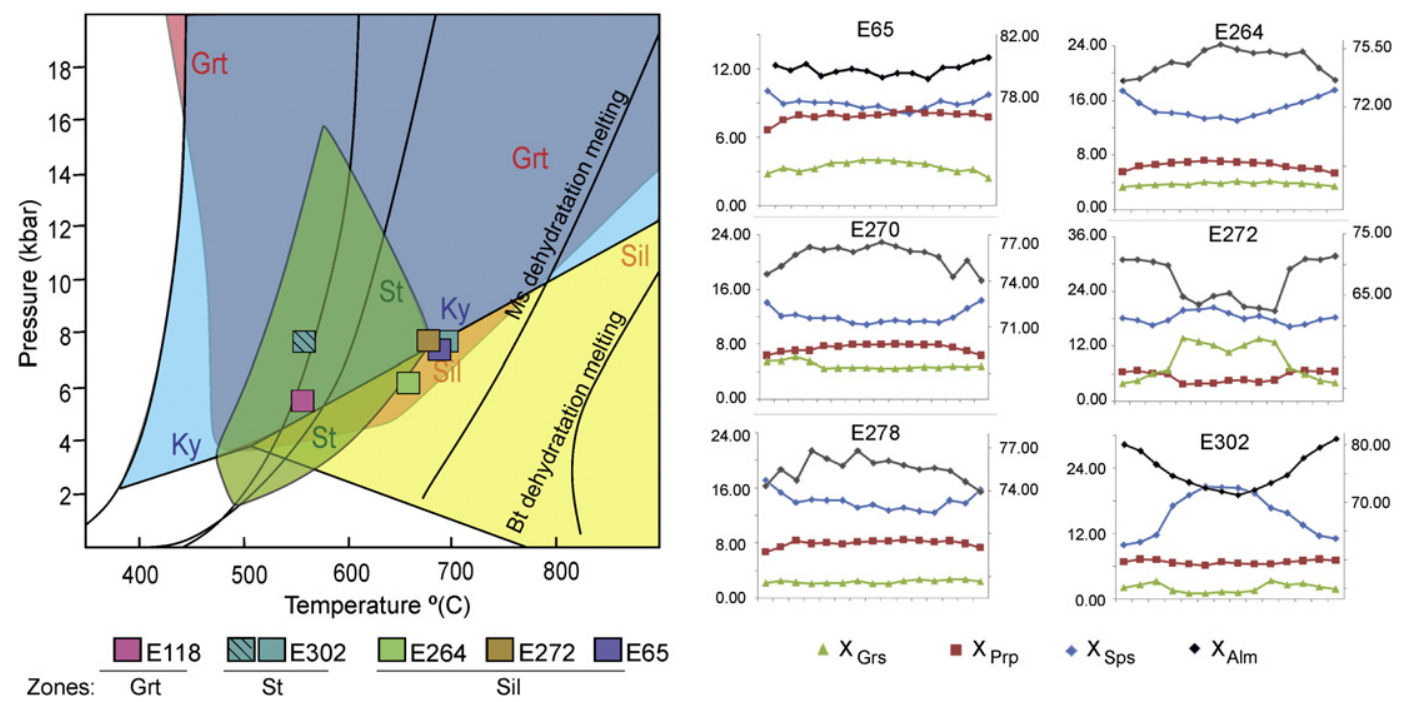

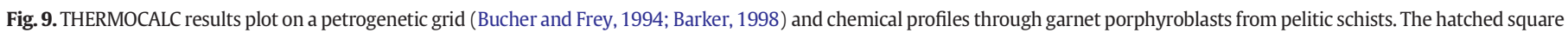
shows conventional thermobarometric data for sample E302. Almandine (Alm) contents are represented at the right axis. 


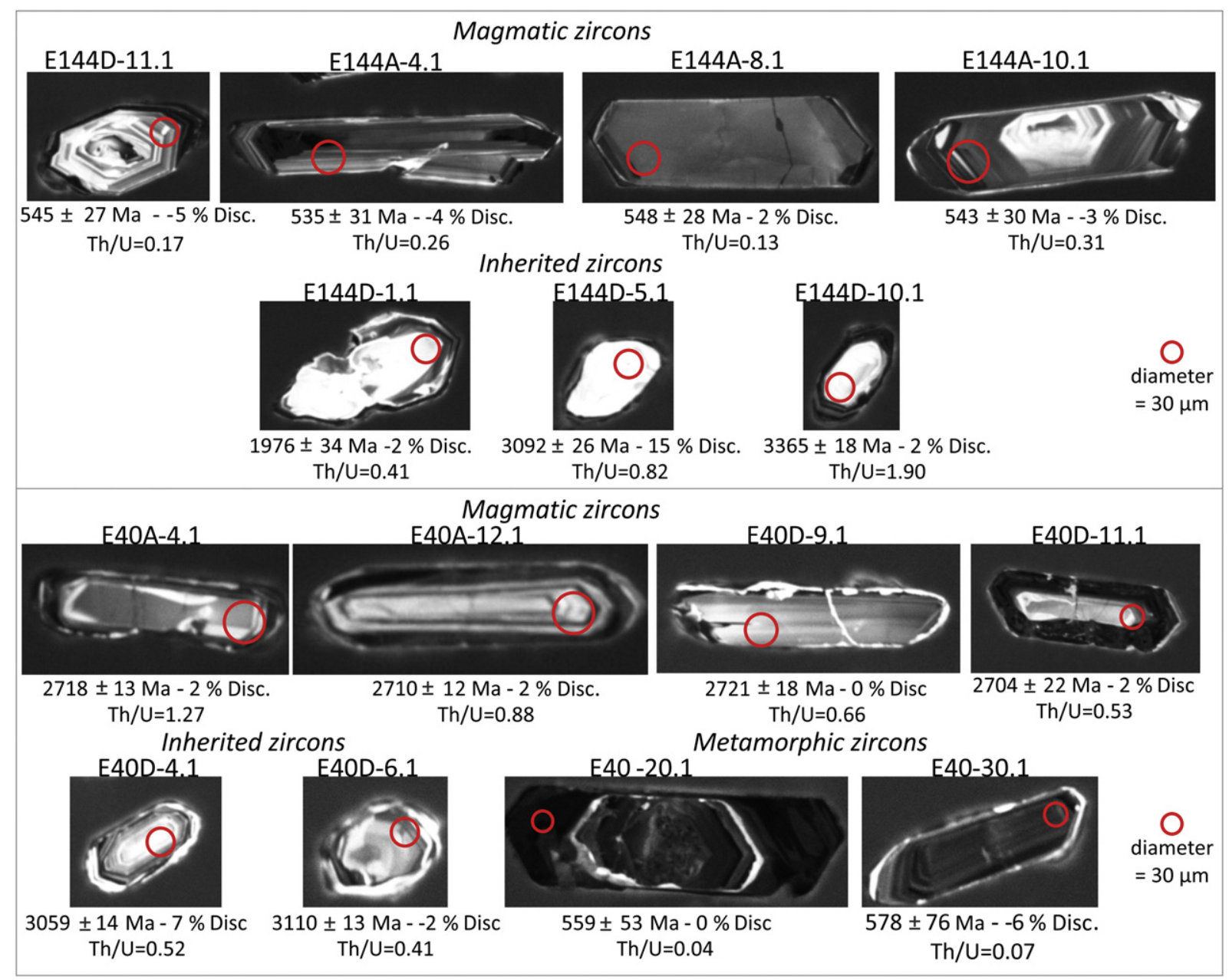

Fig. 10. Cathodoluminescence (CL) images of representative zircon grains from the Guanhães basement gneiss (sample E40) and Santa Rosa granite (sample E144).

(1978). The a-X relationships for minerals were calculated with solid solution adopted by White et al. (2003).

\subsection{Results}

The obtained P-T values are in general agreement with the field metamorphic zoning observed in the schist belt (Table 2, Figs. 4 and 9). Although temperature values given by THERMOCALC and conventional thermometer calculations show some differences, they seem to be coherent for the same sample and in relation to the qualitative evaluations. The descriptions below follows the sample distribution from the base to the top of the Unit B, i.e., from the sillimanite to the garnet zone (Fig. 4).

Samples E264, E270, E272, E278 and E65 are representative of the sillimanite zone, where fibrous sillimanite (fibrolite) is generally abundant and widespread in most outcrops, and can form large (up to $20 \mathrm{~cm}$ ) massive aggregates. However, in thin section this mineral was observed only in sample E278 (among the five collected samples). In this sample, sillimanite occurs as spindle-shaped aggregates of very fine fibrolite, some of them involved by biotite, as an epitaxial overgrowth along the regional foliation $\left(S_{n}\right)$. Pervasively foliated, all samples of the sillimanite zone show garnet poikiloblasts embedded in a fine to coarse-grained muscovite-biotite-quartz-rich matrix. Apatite, tourmaline and opaque minerals are accessory phases in the matrix. Plagioclase compositions vary considerably, ranging from $\mathrm{Ab}_{68}$ to $\mathrm{Ab}_{86}$. $\mathrm{Mg \#}$ $\left(\mathrm{Mg} / \mathrm{Mg}+\mathrm{Fe}^{\mathrm{t}}\right)$ of biotites ranges from 0.45 to 0.52 . Muscovite Mg\# values range from 0.32 to 0.54 .
In the sillimanite zone, garnet crystals vary from anhedral to subhedral and are usually slightly stretched, ranging in size from 1 to $4.2 \mathrm{~mm}$. Randomly or parallel trails of single quartz or Qtz $+\mathrm{Bt}+\mathrm{Ilm}$ (sample E264) or Qtz + Bt + Ilm + Chl + Ms + Tur + Zrn (sample E272) are common inclusion assemblages in garnet crystals. Garnets are usually Alm-rich, with minor spessartite ( $\left.\mathrm{Sps}_{8.1-10.1}\right)$, pyrope $\left(\operatorname{Prp}_{4.0-8.5}\right)$ and grossular $\left(\mathrm{Grs}_{2.1-13.8}\right)$ components (Fig. 9). Analytical results indicate two main chemical patterns. The first, represented by sample E272, involve Sps-rich cores, decreasing toward the borders, and progressively replaced by almandine. These are typical garnets of progressive metamorphism, formed from chlorite breakdown (Deer et al., 1992; Barker, 1998). E272 garnets show rimward increase in Alm + Prp and decrease in Sps + Grs, suggesting overall increase in P-T conditions through time. Garnets from samples E264, E270 and E278 exhibit retrograde patterns, with Alm-rich cores and Sps-rich borders, indicating decreasing P-T conditions through time. Garnets from sample E65 lack evident zoning, suggesting complete chemical homogenization (Fig. 9).

Data for the sillimanite zone schists yielded P-T values in the range of $600-700{ }^{\circ} \mathrm{C}$ at $6-8 \mathrm{kbar}$, corresponding to the intermediate amphibolite facies, with temperatures lower than the muscovite dehydration melting curve. Indeed, no migmatitic feature has been observed in rocks of this zone.

Sample E302, collected in the staurolite zone, shows garnet crystals up to $3 \mathrm{~mm}$ in diameter. Staurolite, locally twinned, occurs as prismatic, subhedral to euhedral poikiloblasts up to $1.2 \mathrm{~cm}$-long, showing quartz inclusions. The small garnet crystals usually show a poikiloblastic texture. 

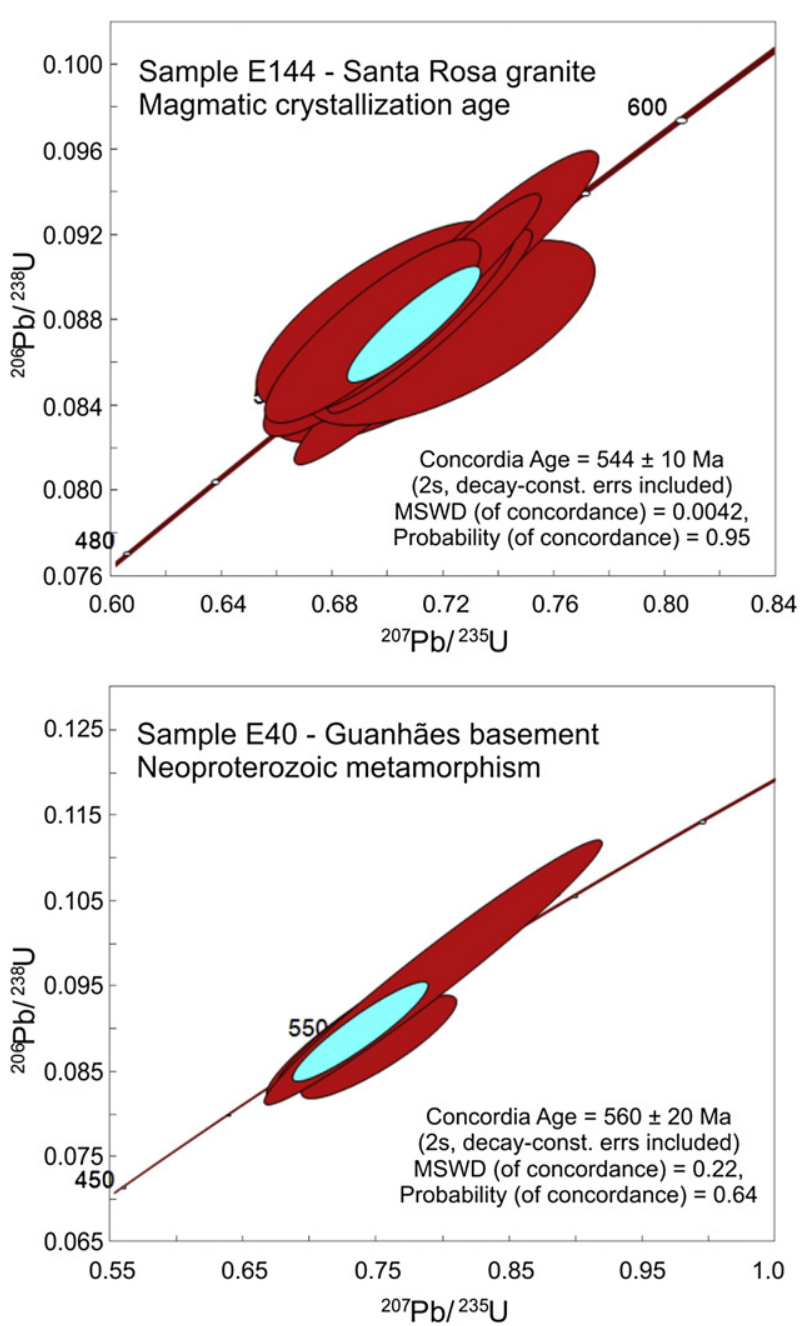
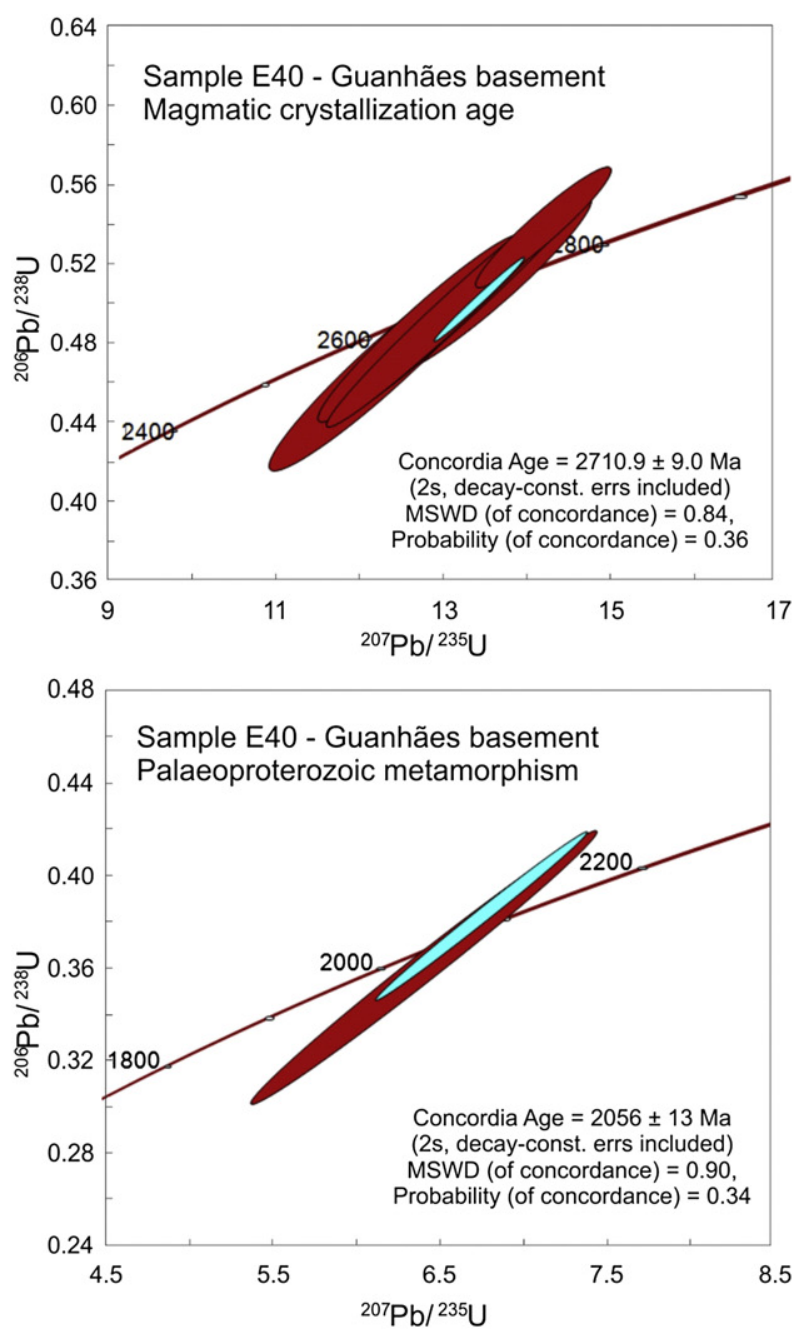

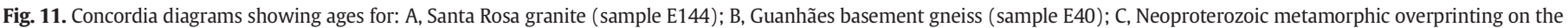
Guanhães basement gneiss; D, Palaeoproterozoic metamorphic overprinting on the Guanhães basement gneiss.

The largest garnet poikiloblasts display a textural zoning that follows the chemical zoning, showing euhedral cores free of inclusions and enrichment in the spessartite molecule $\left(\right.$ Alm $\left._{71.27 \%} \mathrm{Sps}_{20.60 \%} \mathrm{Grs}_{1.11 \%} \operatorname{Prp}_{6.85 \%}\right)$, whereas the borders show skeletal texture and are richer in the almandine component $\left(\mathrm{Alm}_{81.04 \%} \mathrm{Sps}_{9.99 \%} \mathrm{Grs}_{2.12 \%} \mathrm{Prp}_{7.08 \%}\right.$ ), pointing to progressive metamorphism. Biotite $\mathrm{Mg} \#$ are 0.49 to 0.51 . Albite molecule content ranges from $84.4 \%$ to $87.3 \%$. The discrepancies of $\mathrm{T}$ values given by THERMOCALC $\left(705 \pm 36{ }^{\circ} \mathrm{C}\right)$ and conventional methods (c. $550-580{ }^{\circ} \mathrm{C}$ ) can be due to the different number of mineral phases considered in each method.

Sample E118 is an Al-rich calc-silicate rock composed of amphibole poikiloblasts enveloped by a fine-grained granoblastic matrix made up of $\mathrm{Pl}+\mathrm{Qtz}+\mathrm{Czo}+\mathrm{Ep}$. The amphibole is Mg-hornblende, with $\mathrm{Pl}+\mathrm{Qtz}+\mathrm{Ttn}$ inclusions. The epidote is composed of $86 \% \mathrm{Czo}$ and $14 \%$ Ep molecules. Subhedral to euhedral titanite crystals make up to $5 \%$ of the rock. Garnet occurs as fine-grained anhedral and poikiloblastic crystals. Plagioclase shows both andesine and anorthite compositions. The rare garnet poikiloblasts show no chemical zoning and are richer in the Grs component $\left(\mathrm{Alm}_{38.89-43.04 \%} \mathrm{Grs}_{33.33-38.99 \%} \mathrm{Sps}_{19.59-21.21 \%}\right.$ $\operatorname{Prp}_{2.38-2.94 \%}$ ) than the pelitic schists. Garnet Mg\# ranges from 0.0585 to 0.0637 in the calc-silicate rock. This sample yielded the lowest P-T values ( $\mathrm{T}=553 \pm 4{ }^{\circ} \mathrm{\circ}, \mathrm{P}=5.5 \pm 1.1 \mathrm{kbar}$; Table 2, Fig. 9), which correspond to the end of garnet zone in the low amphibolite facies, where chlorite is absent and garnet is generated from biotite breakdown (Barker, 1998).

\section{U-Pb geochronology}

$\mathrm{U}-\mathrm{Pb}$ isotopic analysis was performed by using the Sensitive High Resolution Ion Microprobe (SHRIMP IIe) and Laser Ablasion Multicollector Inductively Coupled Plasma Mass Spectrometry (LAMC-ICP-MS, Neptune Thermo Scientific) equipments of the geochronological laboratories of São Paulo and Brasília universities (Brazil). Rock samples were prepared for analysis in laboratories of São Paulo University and Federal University of Ouro Preto, Brazil.

\subsection{Materials and methods}

Ten to twelve kilograms of fresh samples of a felsic band of an orthogneiss of the Guanhães basement (E40) and from the Santa Rosa foliated granite (E144), and 15 to $20 \mathrm{~kg}$ of fresh to slightly weathered samples from the metasedimentary rocks of the units A and B (E125, E72, E91, E3) were processed for zircon U-Pb analysis. Sample E125 is a medium to coarse-grained garnet-sillimanite-biotite-muscovite schist of the lower portion of Unit A. Sample E72 is a fine to medium-grained quartzite of the upper portion of Unit A. Sample E91 was collected in a medium-grained garnet-muscoviteplagioclase-quartz-biotite schist of the lower portion of Unit B. Sample E3 is a garnet-muscovite-quartz-biotite schist containing thin layers of a fine-grained calc-silicate rock, collected in the upper portion of Unit B. 

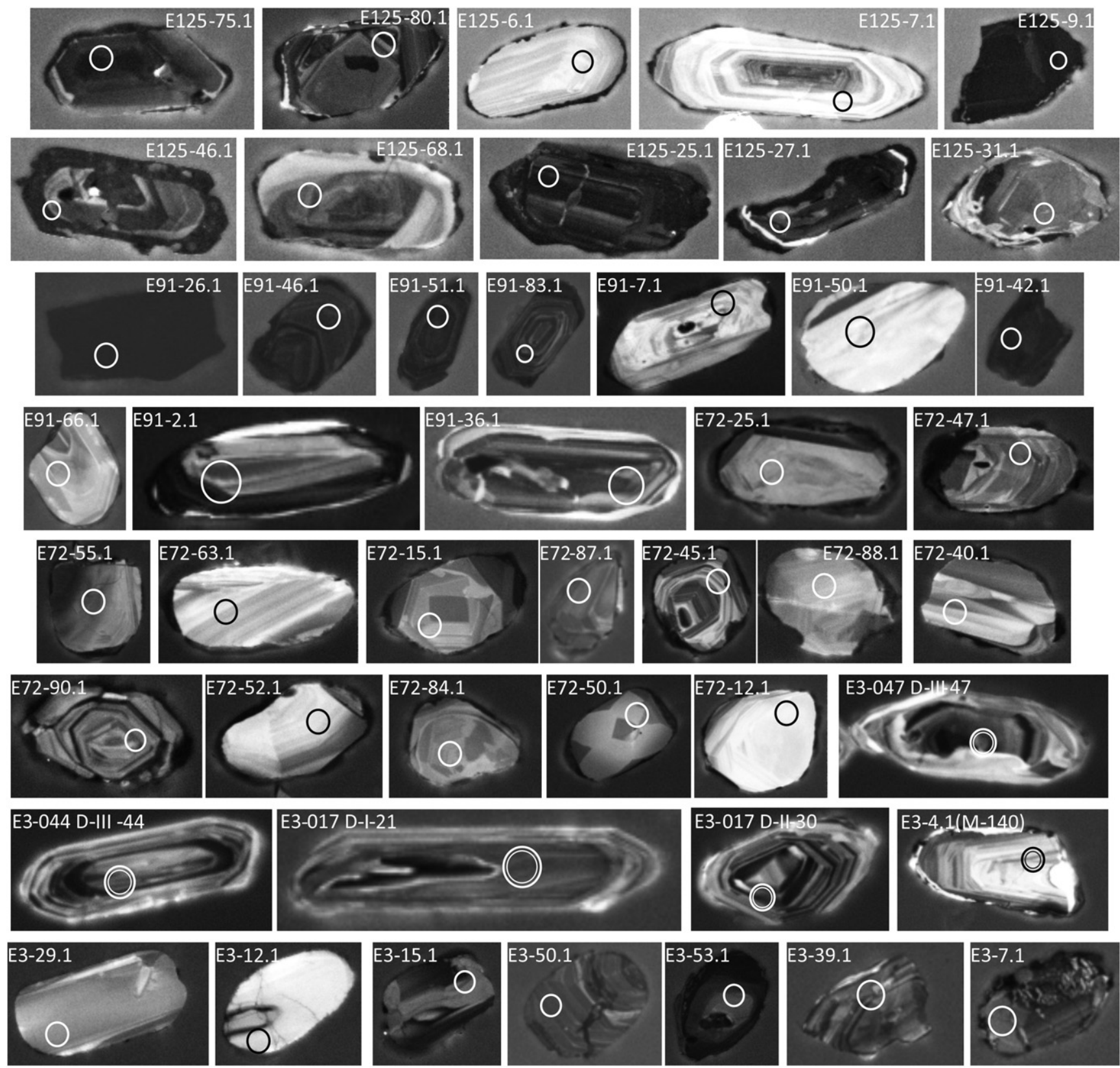

O diameter $=19 \mu \mathrm{m}$
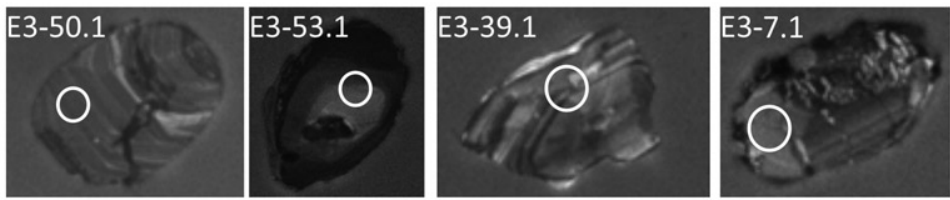

diameter $=25 \mu \mathrm{m}$

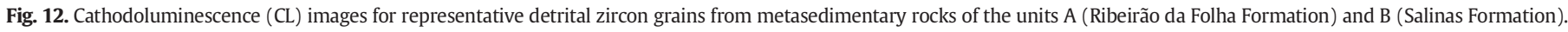

Zircon concentrates were extracted from rock samples by using crushing, grinding, gravimetric and magnetic (Frantz isodynamic separator) techniques for geochronological analysis. Final separation of zircon grains was achieved by hand picking. Only zircon crystals from the least magnetic fractions were selected from samples E40 (Guanhães orthogneiss) and E144 (Santa Rosa granite). For the metasedimentary rocks a number up to 120 detrital grains were randomly recovered from the bulk zircon concentrates from samples E125, E72, E91 and E3. Grains were mounted in epoxy disks and polished to expose their centers. Morphological features and internal structures of zircon grains were revealed by backscattered electron (BSE) and cathodoluminescence (CL) images.

U-Pb (SHRIMP and LA-MC-ICP-MS) analysis were performed on zircon crystals recovered from the Guanhães orthogneiss (E40) and Santa Rosa granite (E144) in order to obtain igneous crystallization and metamorphic ages, as well as to constrain the age of collisional deformation. Analysis on detrital zircon grains from samples of the metasedimentary units A and B (E125, E72, E91, E3) were performed by using U-Pb (LA-MC-ICP-MS) in order to determine maximum depositional ages and sedimentary provenance.

Temora (417 Ma; Black et al., 2003) and GJ-1 standard zircons (Jackson et al., 2004) were used in SHRIMP and LA-MC-ICP-MS analytical routines, respectively. No analytical spot was performed on grain areas with inclusions, fractures and metamict structures. Data reduction used the SQUID software (Ludwig, 2001) for the SHRIMP data, and the Excel sheet developed by Chemale et al. (2012b) for the LA-MC-ICPMS data. For all samples the data from each spot were evaluated taking into account the common $\mathrm{Pb}$ contents, errors of isotopic ratios, percentages of discordance and $\mathrm{Th} / \mathrm{U}$ ratios. From the selected spots only those with discordance lesser than $10 \%$ were used to age calculations for the orthogneiss and granite samples, and plotted in Concordia diagrams. For detrital zircon histograms, not only those spot data with discordance 
generally lesser then $10 \%$ were used but also some grains with discordance lesser than $20 \%$. Concordia diagrams and histograms were obtained with the software Isoplot/Ex (Ludwig, 2003).
The U-Pb analytical data for samples E40, E144, E125, E72, E91 and E3 are available in the following electronic files of supplementary material, respectively: Tab3_U_Pb_zircon_data_sample_E40_Guanhaes_

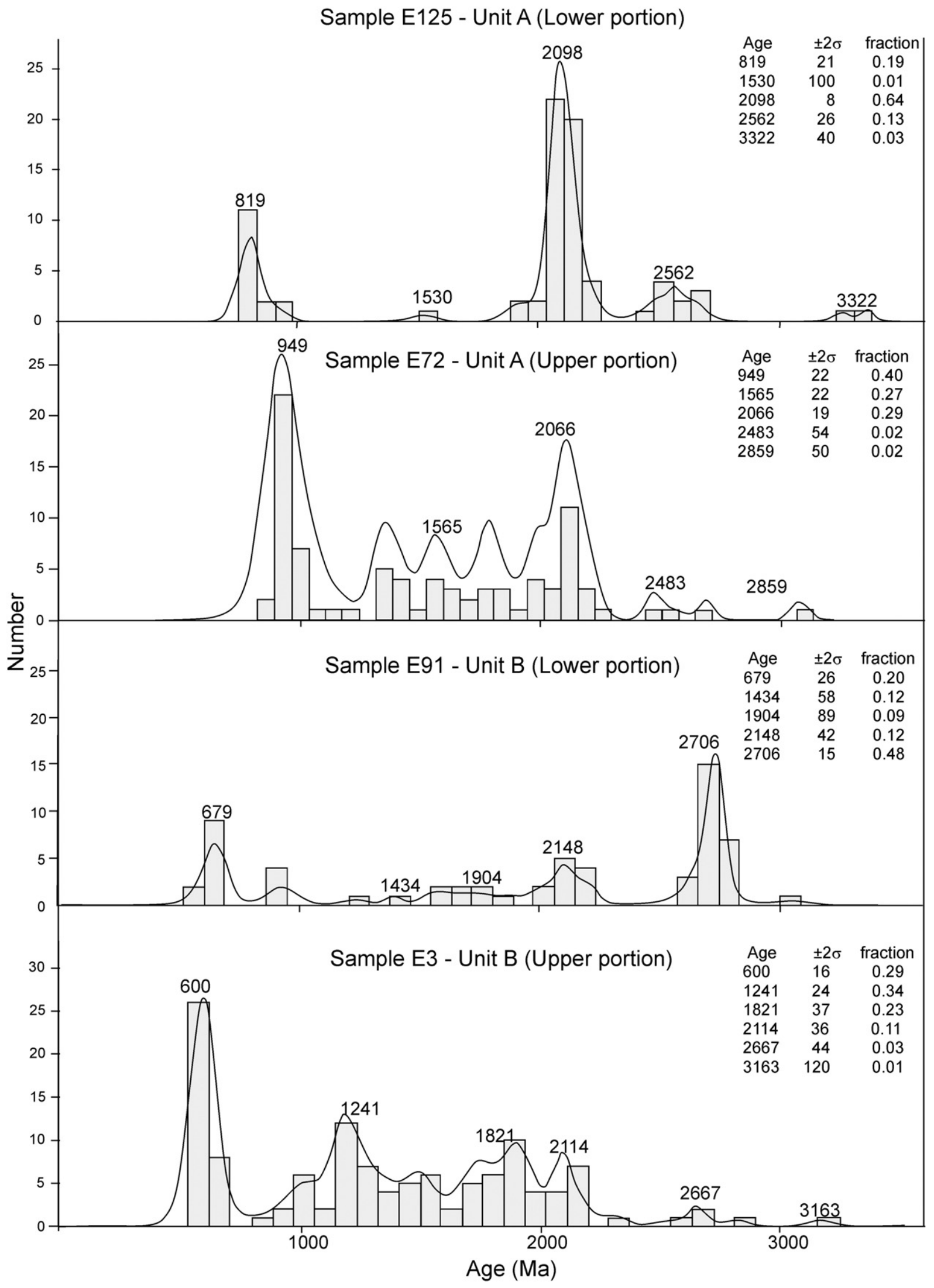

Fig. 13. Frequency histograms showing U-Pb age data for samples from Unit A (E125, E72) and Unit B (E91 and E3). 
Complex.doc, Tab4_U_Pb_zircon_data_sample_E144_Santa_Rosa_ granite.doc, Tab5_U_Pb_zircon_data_sample_E125_unit_A.doc, Tab6_ U_Pb_zircon_data_sample_E72_unit_A.doc, Tab7_U_Pb_zircon_data_ sample_E91_unit_B.doc, Tab8_U_Pb_zircon_data_sample_E3_unit_B.doc.

\section{2. $U-P b$ results for the Guanhães basement gneiss (sample E40)}

Prismatic, subhedral to euhedral, up to $400 \mu \mathrm{m}$ long zircons were extracted from a felsic band of a hornblende-biotite gneiss of the Guanhães basement block, which was strongly affected by the major shear zone close to the contact with the metasedimentary Unit A (Figs. 5 and 10). Oscillatory-zoned domains of zircon crystals yielded the concordant age of $2710 \pm 9 \mathrm{Ma}$, interpreted as the crystallization age for basement gneiss (Figs. 10 and 11). From a group of shorter, subhedral, $200 \mu \mathrm{m}$ long zircon crystals, we have obtained ages around $3100 \mathrm{Ma}$, which have been interpreted as inherited grains.

$\mathrm{U}-\mathrm{Pb}$ data also reveal two metamorphic events imprinted in zircon crystals from this sample of the Guanhães basement block. Two crystals with $\mathrm{Th} / \mathrm{U}$ ratios of 0.12 and 0.18 yielded the metamorphic age of $2056 \pm 13 \mathrm{Ma}$ (Fig. 11). Some other grains exhibit well-defined overgrowth rims with very low $(0.00-0.07) \mathrm{Th} / \mathrm{U}$ ratios (Fig. 10), which yielded a metamorphic age of $560 \pm 20 \mathrm{Ma}$ (Fig. 11).

\section{3. $U-P b$ results for Santa Rosa foliated granite (sample E144)}

The up to $500 \mu \mathrm{m}$ long zircon crystals of this granite are euhedral, prismatic and pyramidal. Inherited grains, which yielded Palaeoproterozoic and Archaean ages, can show rounded borders, and are usually short, ranging from $100 \mu \mathrm{m}$ to $200 \mu \mathrm{m}$ in length (Fig. 10). A magmatic crystallization age of $544 \pm 10$ Ma was obtained for ten zircon crystals extracted from the Santa Rosa foliated granite (Figs. 10 and 11).

\subsection{Detrital zircon $\mathrm{U}-\mathrm{Pb}$ data}

The 90 analyzed grains from sample E125, a schist of the lower Unit A, are prismatic, short to elongated, generally showing rounded terminations and a maximum length of $500 \mu \mathrm{m}$. Some grains are pyramidal with a rounded core. After data reduction, 79 spots could be used for age calculations. The youngest population includes euhedral to subhedral crystals and some rounded grains, whereas older zircon grains mainly show well-rounded shapes (examples shown in Fig. 12, E125-grain spot).

Most (64\%) zircon grains of sample E125 yielded a statistic peak around $2098 \pm 8 \mathrm{Ma}$, representing an age interval of 2.2-2 Ga. Minor Archaean ages point to sources around $2562 \mathrm{Ma}$ and $3332 \mathrm{Ma}$.

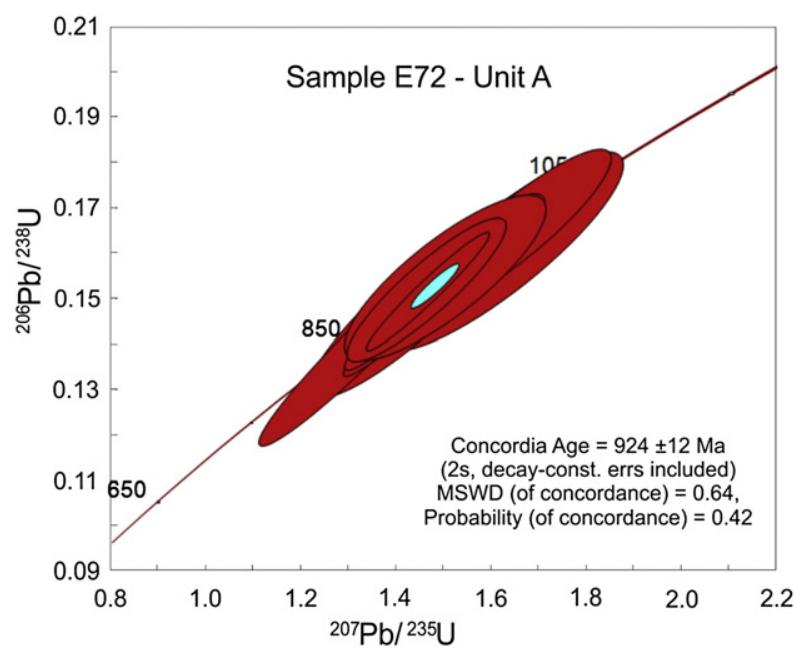

The second most important statistic peak (19\%), of sample E125, representing the zircon population dated from $745 \mathrm{Ma}$ to $926 \mathrm{Ma}$, indicates a maximum deposition age of $819 \pm 21$ Ma for the lower Unit A (Fig. 13).

From sample E72, a quartzite of the upper portion of Unit A, the 87 analyzed grains are invariably short and rounded, anhedral to subhedral, ranging from $80 \mu \mathrm{m}$ to $250 \mu \mathrm{m}$ in length. Rarely, they are thin and elongated with rounded borders (examples shown in Fig. 12). After data reduction and quality check, 86 spot analyses were used for age calculations. Representing $40 \%$ of analyzed zircon grains, the statistic peak around $949 \pm 22$ Ma encompasses the youngest grains of the sample (Fig. 13). Furthermore, 29 grains of this population have yielded the concordant age at $924 \pm 12 \mathrm{Ma}$, which represents a younger component of the source, thus constraining the maximum depositional age of the upper Unit A (Fig. 14). Other important sources of the E72 quartzite are indicated by age peaks at $2066 \pm 19 \mathrm{Ma}(29 \%)$ and $1565 \pm 22 \mathrm{Ma}(27 \%)$.

Two samples from Unit B provided quite different zircon spectra in relation to the samples from Unit A (Fig. 13). From sample E91, a quartz-biotite schist of the lower portion of Unit B, the 81 analyzed grains are generally prismatic with rounded edges, showing lengths from $100 \mu \mathrm{m}$ to $200 \mu \mathrm{m}$ (Fig. 12). After data reduction, 59 spots were used for age calculations. The relative probability diagram shows a main peak at $2704 \pm 15 \mathrm{Ma}$ (40\%), which has been obtained from elongated, subhedral grains with rounded borders. The secondary peaks of $1750 \pm 81 \mathrm{Ma}$ and $2091 \pm 26$ Ma correspond to anhedral, short and rounded zircons. The youngest peak at $677 \pm 23 \mathrm{Ma}(29 \%)$ was yielded by poor-rounded prismatic, subhedral and elongated grains. The youngest age obtained in the same was $580 \pm 35 \mathrm{Ma}$ with $3 \%$ of discordance.

Sample E3, a schist from the upper portion of Unit B, provided 123 zircon grains up to $300 \mu \mathrm{m}$ long (Fig. 12). Euhedral elongated grains with poor-rounded shapes corresponding to $29 \%$ of the population characterizes the youngest peak at $600 \pm 16 \mathrm{Ma}$ (Fig. 13). Moreover, the youngest grains yielded a concordant age of $579 \pm 11$ (Fig. 14), interpreted as the maximum depositional age of the upper Unit B. Rounded, subhedral to anhedral grains comprising $34 \%$ of the whole zircon population have yielded ages around $1241 \pm 24 \mathrm{Ma}$. Other age peaks point to Palaeoproterozoic and Archaean sources.

\section{Discussion and conclusion}

Together, the data we obtained from the detailed field mapping and petrological studies (metamorphism and U-Pb geochronology) support a series of correlations and interpretations discussed bellow.

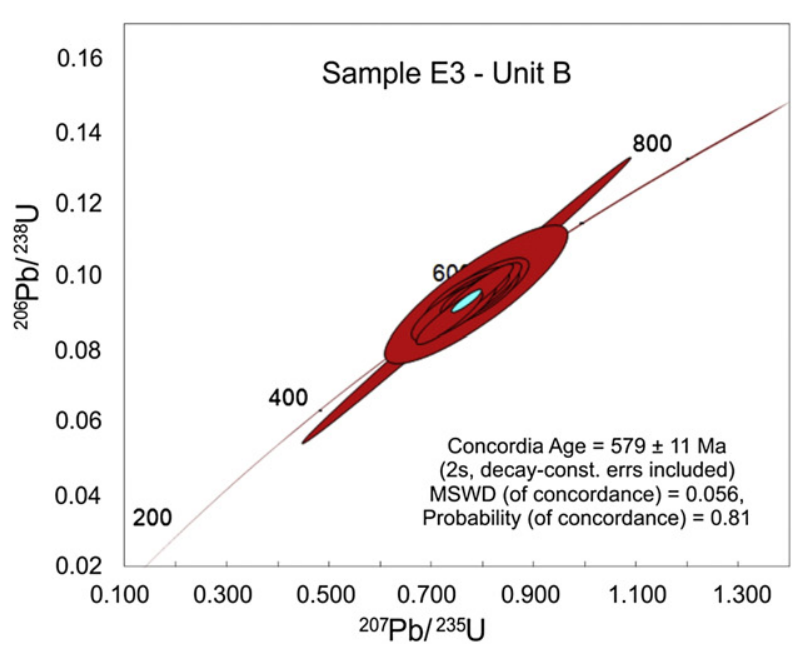

Fig. 14. Concordia diagrams representing ages of the youngest sources for samples E72 (upper Unit A) and E3 (upper Unit B). 
The two metamorphic events $(2056 \pm 13 \mathrm{Ma}$ and $560 \pm 20 \mathrm{Ma})$ detected in zircons from the Archaean Guanhães basement can be ascribed to the Rhyacian-Orosirian orogeny, and the syn-collisional stage of the Brasiliano orogeny, both well documented in the Araçuaî orogen region (Dussin, 1994; Noce et al., 2007; Pedrosa-Soares et al., 2008, 2011b; Silva et al., 2011; Novo, 2013).

Considering the contact relationships and the general characteristics of the Santa Rosa granite, the age obtained for this unit (544 $\pm 10 \mathrm{Ma}$ ) suggests that the syn-collisional stage of the Brasiliano event lasted until the Ediacaran-Cambrian boundary. This value also constrains the minimum age for the studied metasedimentary units around 544 Ma.

$\mathrm{U}-\mathrm{Pb}$ analysis on detrital zircons from Unit A samples show remarkable age peaks around 2.1-2.0 Ga, suggesting that the RhyacianOrosirian magmatic rocks of the Congo-São Francisco palaecontinent provide important sediment sources for this unit. Also, the Archaean ages found in both spectra represent well-known sources in the São Francisco and Congo cratons (e.g., Teixeira et al., 2000; Barbosa and Sabaté, 2004; Lerouge et al., 2006; Noce et al., 2007). Although less important, the Calymmian (c. $1.55 \mathrm{Ga}$ ) age peaks of both samples suggest provenance from the northern Espinhaço basin (Danderfer et al., 2009). The youngest age interval (c. 950-750 Ma) suggest sources related to the development of the precursor basin of the Araçuaí-West Congo orogenic system (Pedrosa-Soares and Alkmim, 2011), namely: i) volcanic and plutonic rocks associated with the Tonian rift represented by the Zadinian and Mayumbian groups of the West Congo belt, and their correlatives in the Araçuaí orogen (Tack et al., 2001; Pedrosa-Soares et al., 2008; Silva et al., 2008; Thiéblemont et al., 2011; Babinski et al., 2012); ii) the Cryogenian anorogenic magmatism recorded by the South Bahia alkaline province of the São Francisco craton (Rosa et al., 2007), the La Louila volcaniclastic rocks (Thiéblemont et al., 2011), and the mafic volcanism associated with the Lower Diamictite Formation of the West Congo belt (Straathof, 2011). Furthermore, the detrital zircon age spectra of Unit A samples are very similar to those published for the Macaúbas Group and correlative units exposed in other domains of the Araçuaí orogen (PedrosaSoares et al., 2000; Martins et al., 2008; Gonçalves-Dias et al., 2011; Babinski et al., 2012).

Taken into account the lithological content, detrital zircon age spectra, maximum depositional ages and sedimentary provenance, Unit A can be thus correlated with the Ribeirão da Folha Formation of the Macaúbas Group (Figs. 3 and 4). As a component of the investigated schist belt it probably represents distal passive margin and oceanic cover deposits, imbricated with ophiolite slivers, as previously suggested by Pedrosa-Soares et al. (1998, 2011a), Aracema et al. (2000) and Queiroga (2010). Furthermore, the minimum sedimentation age for this portion of the Ribeirão da Folha Formation can be constrained by the age of c. $650 \mathrm{Ma}$ for an oceanic plagiogranite (Queiroga et al., 2007; Queiroga, 2010).

The youngest ages yielded by detrital zircons from both samples of the Unit B fall in the interval between c. $640 \mathrm{Ma}$ to c. $580 \mathrm{Ma}$, implying that the Rio Doce magmatic arc (e.g., Nalini et al., 2000; Gonçalves et al., 2010; Pedrosa-Soares et al., 2011b; Novo, 2013; Tedeschi, 2013) acted as an important source for Unit B. As expected, sample E3, collected in the upper part of the Unit B, records the most important contribution from the Rio Doce arc. The other age peaks detected in samples of Unit B reproduce the age spectra of Unit A, which reflect primary sources located in the São Francisco-Congo craton. Unit B can thus be correlated with the Salinas Formation (Figs. 2 and 4), which is portrayed as an orogenic (flysch-type) deposit, accumulated as the Macaúbas passive margin started to interact with the approaching Rio Doce magmatic arc in an early orogenic stage (Lima et al., 2002; Alkmim et al., 2006; Santos et al., 2009). In fact, like the Salinas Formation in its type area, our geochronological data indicate a maximum depositional age of $c$. 579 Ma for Unit B and a significant sedimentary input from the Rio Doce magmatic arc.
The overall structure of the schist belt in the study area resembles an asymmetric, W-verging flower structure bounded by an oblique-slip thrust on the west, and a dextral strike-slip fault system, on the east, which can be viewed as a manifestation of the Abre Campo suture zone (Alkmim et al., 2006). Folds of two generations, thrusts and dextral reverse faults affect the schist belt. All these structures and the typical Barrovian metamorphic zoning reflect the collisional stage of the Araçuaí orogen. According to our results this orogenic stage was in course at around c. $560 \mathrm{Ma}$ and persisted until c. $544 \mathrm{Ma}$, a significantly longer time span than the previously suggested (585-560 Ma) for the syn-collisional stage of the Araçuaí orogen (Pedrosa-Soares et al., 2008, 2011b; Silva et al., 2011). Accordingly, we suggest that the collisional tectonics lasted to c. $544 \mathrm{Ma}$ along the schist belt and, probably, in the whole suture zone.

Our results and correlations suggest that the schist belt, involving deep-sea deposits of the Ribeirão da Folha Formation, tectonic slices of ophiolitic rocks and arc-derived sediments of the Salinas Formation, developed as an accretionary prism according to the following evolutionary model (Fig. 15):

Stage I: As the oceanic portion of the Macaúbas basin enter the subduction zone in the time around or immediately after c. $650 \mathrm{Ma}$, sediments of the Ribeirão da Folha Formation (Unit A) became incorporated in an accretionary wedge, which also included thrust slices of oceanic lithosphere, represented by the metaultramafic rocks in the studied area of the schist belt (Fig. 15a).

Stage II: As the Macaúbas basin closure progressed, its margins began to interact. Turbidites of the Salinas Formation, shed from both the western passive margin and the Rio Doce magmatic arc, filled the space between them and accumulate on top of the accretionary wedge after c. 580 Ma (Fig. 15b).

Stage III: Final closure of the Macaúbas basin, involving the collision of its margins, led to the uplift of this region of the Araçuaí orogen sometime between c. 585 and c. $544 \mathrm{Ma}$. The suturing of the Macaúbas passive margin and Rio Doce arc along the Abre Campo shear zone was associated with intensive dextral transpressional deformation and regional metamorphism (Fig. 15c).

Accretionary wedges locally cover by orogenic deposits (flysch) along suture zones have been documented in several orogenic systems (e.g., Honegger et al., 1982; Hefferan et al., 1992; Huang et al., 1997; Clark and Robertson, 2002; Braid et al., 2010). In two main aspects the investigated schist belt differ from these examples, namely, the absence of structures related to the pre-collisional development of the wedge and the lack of preserved blueschist facies mineral assemblages. In fact, as described in the previous sections all fabric elements documented in the study area seem to reflect the collisional tectonics. Even in the metaultramafic pods and lenses, no structures that could be unquestionably related to their incorporation process into the accretionary wedge have been detected in the study area. The same occurs in other sectors of the schist belt, where ophiolite bodies have been described by Pedrosa-Soares et al. (1998) and Queiroga (2010).

As pointed out by Whitney et al. (2011), preservation of blueschist facies mineral assemblages in orogens depends on a variety of factors. Available data on the Araçuaí orogen indicate that the level of exposure, the geologic architecture and the hot nature of the most part of this orogenic system do not favor the occurrence of high $\mathrm{P}$ low T metamorphic assemblages (Pedrosa-Soares and Noce, 1998; Alkmim et al., 2006).

Diopsidites with similar characteristics and geochemical signature of the São José da Safira diopsidite have been documented in the Cretaceous Oman ophiolite (Python et al., 2007) and Ediacaran- 
A

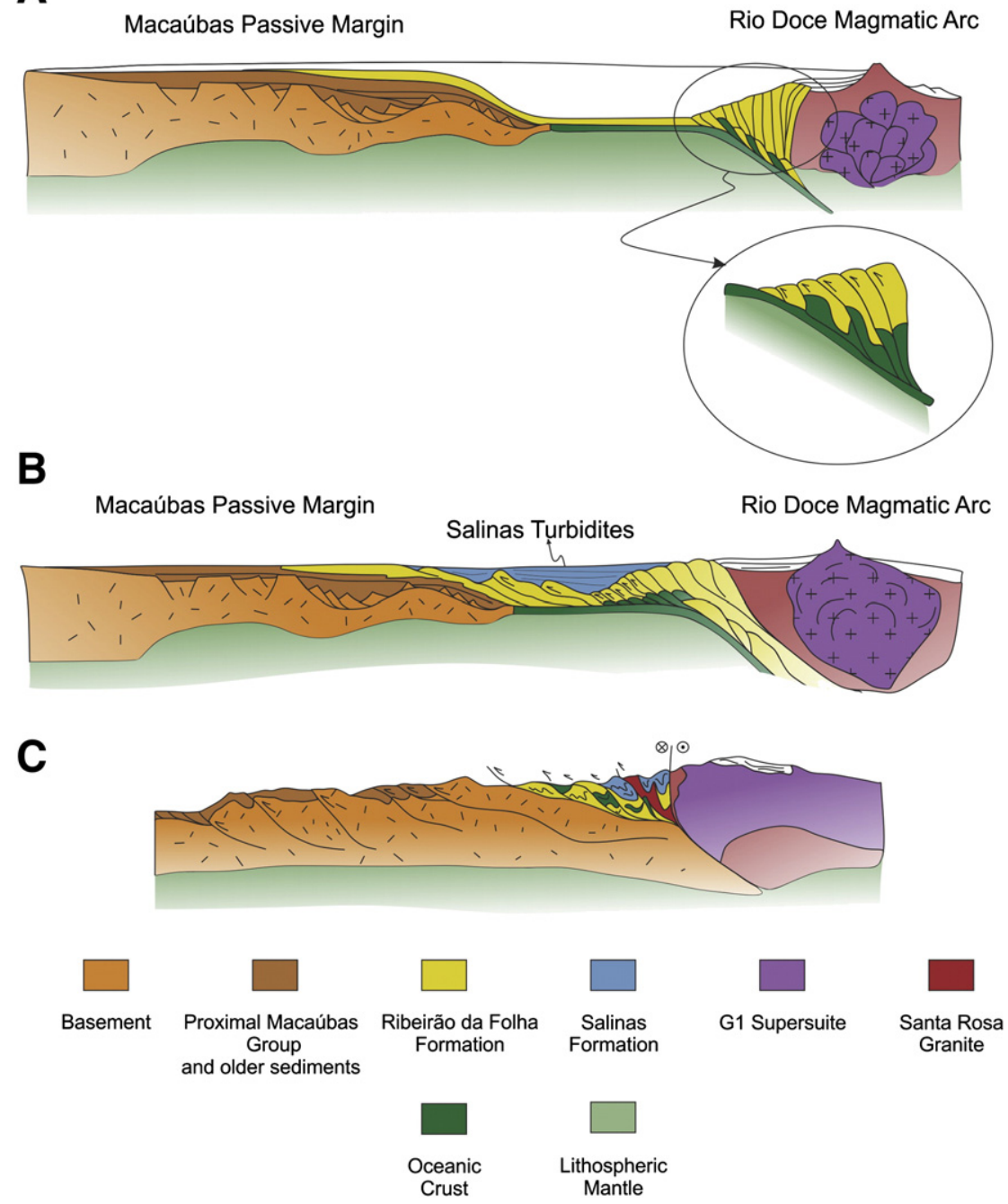

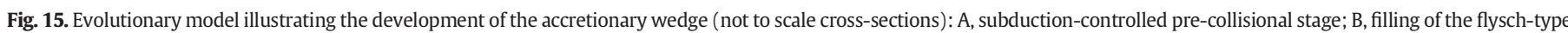
basin (Salinas Formation) in the pre-collisional-collisional transition phase; C, collisional architecture as shown by the present-day rock exposures and landscapes.

Cambrian Palghat-Cauvery suture zone, southern India (Santosh et al., 2010). In both cases, the diopsidites have been interpreted as products of hydrothermal alteration of ultramafic intrusions into upper mantle peridotites. In the second case they were portrayed as components of the sub-oceanic lithospheric mantle, obducted during the final phase of amalgamation of the Gondwana supercontinent, representing Mozambique Ocean closure (Santosh et al., 2010, 2012).

In sum, our results led to the main conclusion that the schist belt preserved along the footwall of the Abre Campo suture zone in the central Araçuaí orogen represents an accretionary wedge, which involves deep marine sediments, slices of the oceanic lithosphere and orogenic (flysch-type) deposits, modified by the collisional tectonics. Indeed, this is an example of accretionary prism formed in a singular orogenic setting, i.e., the confined Araçuaí-West Congo orogenic system which precursor basin was almost completely surrounded by the Congo-São Francisco paleocontinent and represented an inland-sea basin (a large gulf) connected to the Adamastor paleo-ocean (Pedrosa-Soares et al., 1998; Cordani et al., 2003; Goscombe and Gray, 2008; Pedrosa-Soares et al., 2008).

The occurrence of the collisional Santa Rosa granite cutting all units of the accretionary wedge implies that basin closure during the assembly of Western Gondwanaland lasted close to the Ediacaran-Cambrian boundary in the Araçuaí orogen, some 20 Ma after than previously suggested in quoted literature.
Supplementary data to this article can be found online at http:// dx.doi.org/10.1016/j.gr.2013.11.010.

\section{Acknowledgments}

The authors acknowledge financial support provided by Brazilian research and development agencies (CNPq, CAPES, CODEMIG and CPRM) and the staffs of the geochronological laboratories of São Paulo, Brasília and Ouro Preto universities, specially Colombo Tassinari, Miguel Basei, Cristiano Lana, Kei Sato, Maurício de Souza and Vasco dos Loios, and Luis Garcia of the Laboratory of Microanalysis, Federal University of Minas Gerais (LMA-UFMG). Reviews and suggestions provided by Joachim Jacobs, Weronika Gorczyk and Taras Gerya greatly improved the original manuscript.

\section{References}

Alkmim, F.F., Marshak, S., Pedrosa-Soares, A.C., Peres, G.G., Cruz, S.C.P., Whittington, A. 2006. Kinematic evolution of the Aracuaí-West Congo orogen in Brazil and Africa: nutcracker tectonics during the Neoproterozoic assembly of Gondwana. Precambrian Research 149, 43-64.

Almeida, F.F.M., 1977. O Craton do São Francisco. Revista Brasileira de Geociencias 7, 349-364.

Aracema, L.W., Neves, A.C., Ferreira, J.C., Pedrosa-Soares, A.C., Lobato, L.M., Noce, C.M., 2000. Novas evidências de remanescentes oceânicos na Faixa Araçuaí: As rochas meta-ultramáficas de São José da Safira. Geonomos 8, 55-61. 
Babinski, M., Pedrosa-Soares, A.C., Trindade, R.I.F., Martins, M., Noce, C.M., 2012 Neoproterozoic glacial deposits from the Araçuaí orogen, Brazil: age, provenance and correlations with the São Francisco craton and West Congo belt. Gondwana Research 21, 451-465.

Barbosa, J.S.F., Sabaté, P., 2004. Archean and Paleoproterozoic crust of the São Francisco Craton, Bahia, Brazil: geodynamic features. Precambrian Research 133, 1-27.

Barker, A.J., 1998. Introduction to Metamorphic Textures and Microstructures, 2nd ed. Stanley Thornes (Publishers) Ltd, Cheltenham, UK (264 pp.).

Black, L.P., Kamo, S.L., Allen, C.M., Aleinikoff, J.N., Davis, D.W., Korsch, R.J., Foudoulis, C., 2003. TEMORA 1: a new zircon standard for Phanerozoic U-Pb geochronology. Chemical Geology 200, 155-170.

Braid, J.A., Murphy, J.B., Quesada, C., 2010. Structural analysis of an accretionary prism in a continental collisional setting, the Late Paleozoic Pulo do Lobo Zone, Southern Iberia. Gondwana Research 17, 422-439.

Brueckner, H.K., Cunningham, D., Alkmim, F.F., Marshak, S., 2000. Tectonic implications of Precambrian Sm-Nd dates from the southern São Francisco craton and adjacent Araçuaí and Ribeira belts, Brazil. Precambrian Research 99, 255-269.

Bucher, K., Frey, M., 1994. Petrogenesis of Metamorphic rocks, $6^{\mathfrak{a}}$ ed. Springer-Verlag, Berlin (318 pp.).

Chemale, F., Dussin, I.A., Alkmim, F.F., Martins, M.S., Queiroga, G., Armstrong, R., Santos, M.N., 2012a. Unravelling a Proterozoic basin history through detrital zircon geochronology: the case of the Espinhaço Supergroup, Minas Gerais, Brazil. Gondwana Research 22, 200-206.

Chemale, F., Kawashita, K., Dussin, I.A., Ávila, J.N., Justino, D., Bertotti, A., 2012b. U-Pb zircon in situ dating with LA-MC-ICP-MS using a mixed detector configuration. Anais da Academia Brasileira de Ciências 84, 275-295.

Clark, M., Robertson, A., 2002. The role of the Early Tertiary Ulukısla Basin, southern Turkey, in suturing of the Mesozoic Tethys ocean. Journal of the Geological Society 159, 673-690.

Cordani, U.G., D'Agrella-Filho, M.S., Brito-Neves, B.B., Trindade, R.I.F., 2003. Tearing up Rodinia: the Neoproterozoic palaeogeography of South American cratonic fragments. Terra Nova 15, 350-359.

Cunningham, W.D., Marshak, S., Alkmim, F.F., 1996. Structural style of basin inversion at mid-crustal levels: two transects in the internal zone of the Brasiliano Araçuaí Belt Minas Gerais, Brazil. Precambrian Research 77, 1-15.

Cunningham, W.D. Alkmim, F.F., Marshak, S., 1998. A structural transect across the Coastal Mobile belt in the Brazilian Highlands (latitute $20^{\circ} \mathrm{S}$ ): the roots of a Precambrian transpressional orogen. Precambrian Research 92, 251-275.

Danderfer, A., Waele, B., Pedreira, A.J., Nalini, H.A., 2009. New geochronological constraints on the geological evolution of Espinhaço basin within the São Francisco Craton Brazil. Precambrian Research 170, 116-128.

De Campos, C.M., Mendes, J.C., Ludka, I.P., Medeiros, S.R., Moura, J.C., Wallfass, C.A., 2005. Review of the Brasiliano magmatism in Southern Espírito Santo, Brazil, with emphasis on post-collisional magmatism. Journal of the Virtual Explorer (Online) 17, 1-14.

Deer, W.A., Howie, R.A., Zussman, J., 1992. An Introduction to the Rock-Forming Minerals, 2nd edition. Longman Scientific \& Technical, Harlow (696 pp.).

Dussin, I.A., 1994. Evolution structurale de la region de l'Espinhaço meridional, bordure oriental du Craton São Francisco, Brésil: Un exemple des tectoniques superposeés au Protérozoïque. (PhD thesis) Université d'Orléans, France.

Ferry, J.M., Spear, F.S., 1978. Experimental calibration of the partitioning of Fe and Mg between biotite and garnet. Contributions to Mineralogy and Petrology 66, 113-117.

Figueiredo, M.C.H., Campos Neto, M.C., 1993. Geochemistry of the Rio Doce Magmatic Arc, Southeastern Brazil. Anais da Academia Brasileira de Ciências 65, 64-81.

Fischel, D.P., 1998. Geologia e dados isotópicos Sm-Nd do Complexo Mantiqueira e do Cinturão Ribeira na região de Abre Campo, Minas Gerais. (MSc thesis) Universidade de Brasília (98 pp.).

Gonçalves, LE.S., Alkmim, F.F. Pedrosa-Soares, A.C. 2010. Características geoquímicas da Suíte G1, arco magmático do Orógeno Araçuaí, entre Governador Valadares e Ipanema, MG. Revista da Escola de Minas 63, 457-464.

Gonçalves-Dias, T., Pedrosa-Soares, A.C., Dussin, I.A., Alkmim, F.F., Caxito, F.A., Silva, L.C Noce, C.M., 2011. Maximum sedimentation age and provenance of the Jequitinhonh complex in the type-area (Araçuaí orogen): first U-Pb (LA-ICP-MS) data from detrital zircon grains. Geonomos 19, 121-130.

Goscombe, B.D., Gray, D.R., 2008. Structure and strain variation at mid-crustal levels in transpressional orogen: a review of Kaoko Belt structure and the character of West Gondwana amalgamation and dispersal. Gondwana Research 13, 45-85.

Hefferan, K.P., Karson, J.A., Saquaque, A., 1992. Proterozoic collisional basins in a PanAfrican suture zone, Anti-Atlas Mountains, Morocco. Precambrian Research 54 295-319.

Holdoway, M.J., Lee, S.M., 1977. Fe-Mg cordierite stability in high-grade politic rocks based on experimental, theoretical and natural observations. Contribution to Mineralogy and Petrology 63, 175-198.

Honegger, K., Dietrich, V., Frank, W., Gansser, A., Thrni, M., Trommsdorff, V., 1982 Magmatism and metamorphism in the Ladakh Himalayas (the Indus-Tsangpo suture zone). Earth and Planetary Science Letters 60, 253-292.

Huang, C.-Y., Wu, W.-Y., Chang, C.-P., Tsao, S., Yuan, P.B., Lin, C.-W., Kuan-Yuan, X., 1997. Tectonic evolution of accretionary prism in the arc-continent collision terrane of Taiwan. Tectonophysics 281, 31-51.

Jackson, S.E., Pearson, N.J., Griffin, W.L., Belousova, E.A., 2004. The application of laser ablation-inductively coupled plasma-mass spectrometry to in situ U-Pb zircon geochronology. Chemical Geology 211, 47-69.

Lerouge, C., Cocherie, A., Toteu, S.F., Penaye, J., Milési, J.-P., Tchameni, R., Nsifa, E.N., Fanning, M., Deloule, E., 2006. Shrimp U-Pb zircon age evidence for Paleoproterozoic sedimentation and 2.05 syntectonic plutonism in the Nyong Group, South-Western Cameroon: consequences for the Eburnean-Transamazonian belt of NE Brazil and Central Africa. Journal of African Earth Sciences 44, 413-427.
Lima, S.A.A., Martins-Neto, M., Pedrosa-Soares, A.C., Cordani, U.G., Nutman, A., 2002. A Formação Salinas na área tipo, NE de Minas Gerais: uma proposta de revisão da estratigrafia da Faixa Araçuaí com base em evidências sedimentares, metamórficas, e idades U-Pb SHRIMP. Revista Brasileira de Geociencias 32, 491-500.

Ludwig, K.R., 2001. User's manual for Isoplot/Ex Version 2.49 A geochronological toolkit for Microsoft Excel. Berkeley Geochronological Center, Special, Publication $1 \mathrm{a}, 55$.

Ludwig, K.R., 2003. Using Isoplot/Ex, version 3.00, a geochronological toolkit for Microsoft Excel. Berkeley Geochronology Center, Special Publication 1 (43 pp.).

Marshak, S., Alkmim, F.F., Whittington, A., Pedrosa-Soares, A.C., 2006. Extensional collapse in the Neoproterozoic Araçuaí orogen, eastern Brazil: a setting for reactivation of asymmetric crenulation cleavage. Journal Structural Geology 28, 129-147.

Martins, V.T.S., Teixeira, W., Noce, C.M., Pedrosa-Soares, A.C., 2004. Sr and Nd characteristics of Brasiliano-Pan African granitoid plutons of the Araçuaí orogen, southeastern Brazil: tectonic implications. Gondwana Research 7, 75-89.

Martins, M., Karfunkel, J., Noce, C.M., Babinski, M., Pedrosa-Soares, A.C., Sial, A.N., Liu, D., 2008. A sequência pré-glacial do Grupo Macaúbas na área-tipo e o registro da abertura do rifte Araçuaí. Revista Brasileira de Geociencias 38, 768-779.

Nalini, H.A., Bilal, E., Paquette, J.L., Pin, C., Machado, R., 2000. Geochronologie U-Pb et géochimie isotopique $\mathrm{Sr}-\mathrm{Nd}$ des granitoides neoproterozoiques des suites Galileia et Urucum, vallée du Rio Doce, Sud-Est du Brésil. Comptes Rendus de l'Academie de Sciences Paris 331, 459-466.

Neves, A.C., Ferreira, J.C.H., 1999. Mapeamento litoestrutural da Região de São José da Safira. Instituto de Geociências, Universidade Federal de Minas Gerais, Minas Gerais. Trabalho de Graduação (54 pp.).

Noce, C.M., Pedrosa-Soares, A.C., Piuzana, D., Armstrong, R., Laux, J.H., de Campos, C.M., de Medeiros, S.R., 2004. Ages of sedimentation of the kinzigitic complex and of a late orogenic thermal episode in the Araçuaí orogen, Northern Espírito Santo state, Brazil: zircon and monazite U-Pb SHRIMP and ID-TIMS data. Revista Brasileira de Geociencias 34, 587-592.

Noce, C.M., Pedrosa-Soares, A.C., Silva, L.C., Armstrong, R., Piuzana, D., 2007. Evolution of polyciclic basement complexes in the Araçuaíorogen, based on U-Pb SHRIMP data: implications for Brazil-Africa links in Paleoproterozoic time. Precambrian Research $159,60-78$

Novo, T.A., 2013. Caracterização do Complexo Pocrane, magmatismo básico mesoproterozóico e unidade neoproterozóicas do sistema Araçuaí-Ribeira, com ênfase em geocronologia U-Pb (SHRIMP e LA-ICP-MS). (PhD Thesis) Universidade Federal de Minas Gerais, Brazil.

Novo, T., Pedrosa-Soares, A.C., Noce, C.M., Alkmim, F.F., Dussin, I., 2010. Rochas charnockíticas do sudeste de Minas Gerais: a raiz granulítica do arco Magmático do Orógeno Araçuaí. Revista Brasileira de Geociencias 40, 573-592.

Pedrosa-Soares, A.C., Alkmim, F.F., 2011. How many rifting events preceded the development of the Aracuaí-West Congo orogen? Geonomos 19, 244-251.

Pedrosa-Soares, A.C., Noce, C.M., 1998. Where is the suture zone of the Neoproterozoic Araçuaí-West Congo orogen? 14th International Conference on Basement TectonicsUniversidade Federal de Ouro Preto 35-37 (Abstracts).

Pedrosa-Soares, A.C., Noce, C.M., Vidal, P., Monteiro, R.L.B.P., Leonardos, O.H., 1992. Toward a new tectonic model for the Late Proterozoic Araçuaí (SE Brazil)-West Congolian (SW Africa) Belt. Journal of South American Earth Sciences 6, 33-47.

Pedrosa-Soares, A.C., Vidal, P., Leonardos, O.H., Brito-Neves, B.B., 1998. Neoproterozoic oceanic remnants in eastern Brazil: further evidence and refutation of an exclusively ensialic evolution for the Araçuaí-West Congo Orogen. Geology 26, 519-522.

Pedrosa-Soares, A.C., Cordani, U.G., Nutman, A., 2000. Constraining the age of Neoproterozoic glaciation in eastern Brazil: first U-Pb (SHRIMP) data from detrital zircons. Revista Brasileira de Geociencias 30, 58-61.

Pedrosa-Soares, A.C., Noce, C.M., Wiedemann, C.M., Pinto, C.P., 2001. The Araçuaí-West Congo Orogen in Brazil: an overview of a confined orogen formed during Gondwanaland assembly. Precambrian Research 110, 307-323.

Pedrosa-Soares, A.C., Noce, C.M., Alkmim, F.F., Silva, L.C., Babinski, M., Cordani, U., Castañeda, C., 2007. Orógeno Araçuaí: síntese do conhecimento 30 anos após Almeida 1977. Geonomos 15, 1-16.

Pedrosa-Soares, A.C., Alkmim, F.F., Tack, L., Noce, C.M., Babinski, M., Silva, L.C., MartinsNeto, M., 2008. Similarities and differences between the Brazilian and African counterparts of the Neoproterozoic Araçuaí-West Congo Orogen. In: Pankhurst, J.R., Trouw, R.A.J., Brito Neves, B.B., De Wit, M.J. (Eds.), West Gondwana: Pre-Cenozoic Correlations across the South Atlantic Region. Geological Society, London, Special Publications, 294, pp. 153-172.

Pedrosa-Soares, A.C., Babinski, M., Noce, C., Martins, M., Queiroga, G., Vilela, F., 2011a. The Neoproterozoic Macaúbas Group (Araçuaí orogen, SE Brazil) with emphasis on the diamictite formations. In: Arnaud, E., Halverson, G.P., Shields-Zhou, G. (Eds.), The Geological Record of Neoproterozoic Glaciations. Geological Society, London, Memoirs, 36, pp. 523-534

Pedrosa-Soares, A.C., De Campos, C.P., Noce, C., Silva, L.C., Novo, T., Roncato, R., Medeiros, S., Castañeda, C., Queiroga, G., Dantas, E., Dussin, I., Alkmim, F., 2011b. Late Neoproterozoic-Cambrian granitic magmatism in the Araçuaí orogen (Brazil), the Eastern Brazilian Pegmatite Province and related mineral resources. Geological Society, London, Special Publications 350, 25-51.

Peixoto, E., 2013. Caracterização estratigráfica, tectônica, metamórfica e geocronológica do Orógeno Araçuaí na região de São José da Safira-Virgolândia, Minas Gerais. (MSc thesis) Programa de Pós-Graduação em Geologia.Universidade Federal de Minas Gerais, Belo Horizonte, Brazil.

Peres, G.G. Alkmim, F.F. Jordt-Evangelista, H., 2004. The southern Araçuaí belt and the DomSilvério Group: geologic architecture and tectonic significance. Anais Academia Brasileira de Ciências 76, 771-790.

Pinto, C.P., Drumond, J.B.V., Féboli, W.L. (coord.) 2000. Projeto Leste, Etapas 1 e 2. CPRMCOMIG, Belo Horizonte, CD-ROM. 
Powell, R., Holland, T.J.B., 2008. On thermobarometry. Journal of Metamorphic Geology $26,155-179$

Powell, R., Holland, T.J.B., Worley, B., 1998. Calculating phase diagrams involving solid solutions via non-linear equations, with examples using THERMOCALC. Journal of Metamorphic Geology 16, 577-588.

Python, M., Ceuleneer, G., Ishida, Y., Barrat, J.-A., Arai, S., 2007. Oman diopsidites: a new lithology diagnostic of very high temperature hydrothermal circulation in mantle peridotite below oceanic spreading centres. Earth and Planetary Science Letters 255, 289-309.

Queiroga, G.N., 2010. Caracterização de restos de litosfera oceânica do Orógeno Araçuaî entre os paralelos $17^{\circ}$ e $21^{\circ} \mathrm{S}$. (PhD thesis) Universidade Federal de Minas Gerais, Brazil.

Queiroga, G.N., Pedrosa-Soares, A.C., Quéméneur, J., Castañeda, C., 2006. A unidade metassedimentar do ofiolito de Ribeirão da Folha, Orógeno Araçuaí, Minas Gerais: petrografia, geotermobarometria e calcografia. Geonomos 14, 25-35.

Queiroga, G., Pedrosa-Soares, A.C., Noce, C.M., Alkmim, F.F., Pimentel, M.M., Dantas, E., Martins, M., Castañeda, C., Suita, M.T.F., Prichard, F., 2007. Age of the Ribeirão da Folha ophiolite, Araçuaí Orogen: the U-Pb zircon dating of a plagiogranite. Geonomos 15, 61-65.

Rosa, M.L.S., Conceição, H., Macambira, M., Galarza, M.C., Cunha, M., Menezes, R., Marinho, M.M., Cruz-Filho, B.E., Rios, D.C., 2007. Neoproterozoic anorogenic magmatism in the Southern Bahia Alkaline Province of NE Brazil: $\mathrm{U}-\mathrm{Pb}$ and $\mathrm{Pb}-\mathrm{Pb}$ ages of the blue sodalite syenites. Lithos 97, 88-97.

Santos, R.F., Alkmim, F.F., Pedrosa-Soares, A.C., 2009. A Formação Salinas, Orógeno Araçuaí, MG: História deformacional e significado tectônico. Revista Brasileira de Geociencias 39, 81-100.

Santosh, M., Rajesh, V.J., Tsunogae, T., Arai, S., 2010. Diopsidites from a NeoproterozoicCambrian suture in southern India. Geological Magazine 147, 777-788.

Santosh, M., Xiao, W.J., Tsunogae, T., Chetty, T.R.K., Yellappa, T., 2012. The Neoproterozoic subduction complex in southern India: SIMS zircon U-Pb ages and implications for Gondwana assembly. Precambrian Research 192-195, 190-208.

Silva, S. L., 2000. Folha Santa Maria do Suaçuí (SE.23-Z-B-III). Escala 1:100.000. In: Projeto Leste. Belo Horizonte: SEME/COMIG/CPRM. CD-ROM.

Silva, L.C., Pedrosa-Soares, A.C., Teixeira, L.R., Armstrong, R., 2008. Tonian rift-related, A-type continental plutonism in the Araçuaí Orogen, eastern Brazil: new evidence for the breakup stage of the São Francisco-Congo Paleocontinent. Gondwana Research 13, 527-537.
Silva, L.C., Pedrosa-Soares, A.C., Armstrong, R., Noce, C.M., 2011. Determining the timing of the collisional period of the Araçuaí Orogen by using high resolution U-Pb geochronology on zircon: a contribution to the history of western Gondwana amalgamation. Geonomos 19, 180-197.

Straathof, G.B., 2011. Neoproterozoic Low Latitude Glaciations: An African Perspective. (PhD thesis) University of Edinburgh, Scotland, UK.

Tack, L., Wingate, M.T., Liégeois, J.-P., Fernandez-Alonso, M., Deblond, A., 2001. Early Neoproterozoic magmatism (1000-910 Ma) of the Zadinian and Mayumbian groups (Bas-Congo): onset of Rodinian rifting at the western edge of the Congo craton. Precambrian Research 110, 277-306.

Tedeschi, M., 2013. Caracterização do arco magmático do Orógeno Araçuaí entre Frei Inocêncio e Itambacuri, Minas Gerais. (MSc thesis) Programa de Pós-Graduação em Geologia.Universidade Federal de Minas Gerais, Belo Horizonte, Brazil.

Teixeira, W., Sabaté, P., Barbosa, J., Noce, C.M., Carneiro, M.A., 2000. Archean and Paleoproterozoic tectonic evolution of the São Francisco Craton. In: Cordani, U.G. Milani, E.J., Thomaz-Filho, A., Campos, D.A. (Eds.), Tectonic Evolution of South America, Rio de Janeiro. 31st International Geological Congress, pp. 101-138.

Thiéblemont, D., Prian, J.P., Goujou, J.C., Boulingui, B., Ekogha, H., Kassadou, A.B., SimoNdounze, S., Walemba, A., Préat, A., Theunissen, K., Cocherie, A., Guerrot, C., 2011. Timing and characteristics of Neoproterozoic magmatism in SW-Gabon: first geochronogical and geochemical data on the West-Congolian orogen in Gabon (SYSMIN project, Gabon 2005-2009). 23 CAG-Colloquium of African Geology, posters and abstracts.

Thompson, A.B., 1976. Mineral reactions in pelitic rocks: II.Calculation of some P-T-X (Fe-Mg) phase relations. American Journal of Science 276, 425-454.

Trompette, R., 1994. Geology of Western Gondwana (2000-500 Ma). Pan-AfricanBrasiliano aggregation of South America and Africa.A.A. Balkema, Rotterdam (350 pp.).

Uhlein, A., Trompette, R., Alvarenga, C., 1999. Neoproterozoic glacial and gravitational sedimentation on a continental rifted margin: the Jequitaí-Macaúbas sequence (Minas Gerais, Brazil). Journal of South American Earth Sciences 12, 435-451.

Vieira, V.S., 2007. Significado do Grupo Rio Doce no Contexto do Orógeno Araçuaí. Belo Horizonte. (Ph.D. thesis) Universidade Federal de Minas Gerais.

White, R., Powell, R., Clarke, G., 2003. Prograde metamorphic assemblage evolution during partial melting of metasedimentary rocks at low pressures: migmatites from Mt. Stafford, Central Australia. Journal of Petrology 44, 1937-1960.

Whitney, D.L., Teyssier, C., Toraman, E., Seaton, N.C.A., Fayon, A.K., 2011. Metamorphic and tectonic evolution of a structurally continuous blueschist-to-Barrovian terrane, Sivrihisar Massif, Turkey. Journal of Metamorphic Geology 29, 193-212. 